\title{
Application of a dc Fano effect polarized electron source to low-energy electron-atom scattering
}

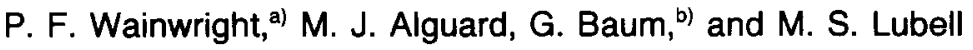 \\ J. W. Gibbs Laboratory, Yale University, New Haven, Connecticut 06520 \\ (Received 19 January 1978; in final form, 3 February 1978)
}

\begin{abstract}
A polarized electron source based upon the photoionization of unpolarized Cs atoms by circularly polarized light (Fano effect) has been developed and applied to the study of spin dependence in low-energy electron-atom scattering. Electron intensities of $10 \mathrm{nA}$ with polarizations of $0.63 \pm 0.03$ have been obtained routinely during continuous runs of up to $75 \mathrm{~h}$. Frequent optical reversal of the direction of the longitudinal electron polarization minimizes systematic effects so that helicity dependent electron-scattering asymmetries smaller than $4 \times 10^{-4}$ can be measured.
\end{abstract}

\section{INTRODUCTION}

\section{A. Survey}

In recent years there has been increasing interest in the spin dependence in electron-atom collisions, with the relationship between theory and experiment being truly symbiotic. In the case of electron-alkali collisions, the early optical pumping measurements of spin-flip cross sections in $\mathrm{Na}, \mathrm{Rb}$, and $\mathrm{Cs}$ at thermal energies ${ }^{1-13}$ have been augmented by detailed crossed-beam studies of elastic and inelastic scattering of electrons by $\mathrm{Li}, \mathrm{K}, \mathrm{Na}$, and $\mathrm{Rb}$. These differential scattering measurements, covering an energy range from several tenths of an $\mathrm{eV}$ to several $\mathrm{eV}$, have incorporated polarized atomic beams with polarization analysis of either the recoil atom ${ }^{4-8}$ or the scattered electron. ${ }^{9}$ An electron trap with a polarized atomic beam and an electron polarization analyzer has also been used to study the total spin-exchange cross section in $\mathrm{e}-\mathrm{K}$ scattering at $\mathrm{eV}$ energies. ${ }^{10}$

Progress in the theoretical understanding of the spin dependence in electron-alkali scattering has also been substantial. Following the calculations of Stone and Reintz, ${ }^{11}$ Garrett and Mann ${ }^{12,13}$ and Garrett, ${ }^{14}$ who first explicitly demonstrated the importance of spin exchange, a number of more accurate theoretical studies have been carried out. ${ }^{15-23}$ Several of these studies have used relativistic formulations which include the spinorbit as well as the spin-exchange interactions. ${ }^{19,20,22,23}$ The effect of the spin-orbit interaction, alone, on electron scattering from $\mathrm{Hg}$ and $\mathrm{Au}$ is well known from experimental and theoretical studies of Mott scattering at both high and low energies, and has been discussed at length in a number of review articles. ${ }^{24-27}$ Pronounced spin-orbit effects were also predicted ${ }^{28}$ and have been observed $^{29,30}$ in resonance scattering from neon. Only in the case of electron-alkali scattering, however, has the combination of spin-orbit and spin-exchange effects been considered. ${ }^{23}$

In electron-hydrogen scattering, on the other hand, the effect of the spin-orbit interaction essentially can be neglected, and only the nonrelativistic wave equation need be considered. Because of the relative simplicity of the interaction, the electron-hydrogen problem has undergone the greatest theoretical scrutiny as a test of the approximation methods which have been employed to describe electron-atom collisions. Theoretical activity has been so great in recent years that a complete list of references is prohibitive. Summaries of and references to various calculations can usually be found in the longer theoretical papers and in reviews. ${ }^{31-35}$ At low energies ( $\$ 30 \mathrm{eV}$ ), variational techniques ${ }^{32,35-41}$ have been quite successful in reproducing spin-averaged elastic differential cross sections and resonances. ${ }^{42} \mathrm{At}$ high energies ( $\geq 100 \mathrm{eV}$ ), Born, eikonal, and Glauber elastic scattering calculations s3-46 $^{40}$ in fair agreement with experimental observation. ${ }^{42}$ In the intermediate energy region, between 30 and $100 \mathrm{eV}$, calculations are generally less reliable.

While the large majority of theoretical calculations have taken the exchange interaction into account, until recently no experiments had been reported which were able to provide information about the direct and exchange cross sections in elastic electron-hydrogen scattering. ${ }^{42,47}$ In fact, the only electron-hydrogen collision channel for which any exchange scattering experimental data had been obtained was the $1 \mathrm{~s}-2 \mathrm{~s}$ inelastic excitation channel. ${ }^{48}$ Unfortunately the theoretical understanding of inelastic scattering, as well as of ionization, has not progressed as far as that of elastic scattering.

In light of the apparent growing interest in spin effects in electron-atom collisions, several papers have been published which provide prescriptions for the analysis of experiments employing spin-polarized sources of atoms and electrons and spin-polarization analyzers. ${ }^{49-52}$ Until very recently however, only one experiment, the study of exchange excitation in $\mathrm{Hg}$, had been carried out using polarized electrons as the incident beam. ${ }^{53-55}$ Adequate sources of polarized electrons simply have not been available for more fundamental studies.

Over the course of the last ten years the progress in the field of polarized-electron atomic physics has 
been delineated in a series of review articles and monographs ${ }^{7.24-27.56-58}$ covering both the development and application of polarized electron sources. In this paper we report on a major advance in the development of polarized electron sources and on its application to the study of electron-hydrogen scattering.

\section{B. Polarization experiments}

In the absence of the spin-orbit interaction, the scattering of electrons by one-electron atoms can be described by the nonrelativistic wave equation

$$
\left[\mathscr{H}_{0}\left(\mathbf{r}_{1}\right)+\mathscr{H}_{a}\left(\mathbf{r}_{2}\right)+V\left(\mathbf{r}_{1}, \mathbf{r}_{2}\right)-E\right] \Psi\left(\mathbf{r}_{1} s_{1}, \mathbf{r}_{2} s_{2}\right)=0,
$$

where $\mathbf{r}_{1}$ and $\mathbf{r}_{2}$ are the position coordinates of the incident and atomic electrons, respectively, $\mathscr{H}_{0}$ and $\mathscr{H}_{1 t}$ are the Hamiltonians of the incident and atomic electrons, respectively, $V$ is the interaction potential, $E$ is the total energy, and $s_{1}$ and $s_{2}$ are the spin coordinates of the incident and atomic electrons, respectively. Fermi-Dirac statistics require that the wave function $\Psi\left(\mathbf{r}_{1} s_{1}, \mathbf{r}_{2} s_{2}\right)$ be totally antisymmetric under particle exchange. Since the total Hamiltonian is independent of electron spin and is furthermore symmetric under particle exchange, it is easily seen that the total wave function can be written as a product of a position wave function, $\psi\left(\mathbf{r}_{1}, \mathbf{r}_{2}\right)$, and a spinor, $\chi\left(s_{1}, s_{2}\right)$, and that $\psi\left(\mathbf{r}_{1}, \mathbf{r}_{2}\right)$ and $\chi\left(s_{1}, s_{2}\right)$ must separately possess symmetry properties under particle interchange. The symmetric position function, $\psi_{+}\left(\mathbf{r}_{1}, \mathbf{r}_{2}\right)$, is then paired with the antisymmetric singlet spinor, $\chi(S=0)$, and the antisymmetric position function, $\psi_{-}\left(\mathbf{r}_{1}, \mathbf{r}_{2}\right)$, is paired with the symmetric triplet spinors, $\chi(S=1)$, where $S$ is the total spin of the two-electron system.

The asymptotic forms of $\psi_{ \pm}\left(\mathbf{r}_{1}, \mathbf{r}_{2}\right)$ are given by

$$
\begin{aligned}
\psi_{ \pm}\left(r_{1}, r_{2}\right) \underset{r_{1} \rightarrow \infty}{\rightarrow} e^{i \mathbf{k} \cdot \mathbf{r}_{1}} \phi_{0}\left(\mathbf{r}_{2}\right) \\
\quad+\sum_{\gamma} \frac{e^{i k^{\prime} r_{1}}}{r_{1}}\left[f_{0 \gamma}\left(\hat{k}, \hat{k}^{\prime}\right) \pm g_{0 \gamma}\left(\hat{k}, \hat{k}^{\prime}\right)\right] \phi_{\gamma}\left(\mathbf{r}_{2}\right)
\end{aligned}
$$

where $\mathbf{k}$ and $\mathbf{k}^{\prime}$ are, respectively, the momenta (in atomic units) of the incident and scattered electron, $\phi_{0}\left(\mathbf{r}_{2}\right)$ and $\phi_{\gamma}\left(\mathbf{r}_{2}\right)$ are, respectively, the initial and final state atomic wave functions, and $f_{0 \gamma}\left(\hat{k}, \hat{k}^{\prime}\right)$ and $g_{0 \gamma}\left(\hat{k}, \hat{k}^{\prime}\right)$ are, respectively, the "direct" and "exchange" scattering amplitudes. Thus singlet scattering is characterized by the amplitude $(f+g)$, and triplet scattering by the amplitude $(f-g)$, where the subscripts for scattering into a particular channel $\left(f_{00} \pm g_{00}\right.$ for elastic scattering, for example) are implicit.

Since $f$ and $g$ are complex quantities, three independent parameters are necessary for the description of the scattering into any one channel. As an example, the magnitude of $f$, the magnitude of $g$, and their relative phase must be specified, with the overall phase being an arbitrary, physically inaccessible quantity. For experiments with unpolarized particles only the weighted sum of the singlet and triplet cross sections can be
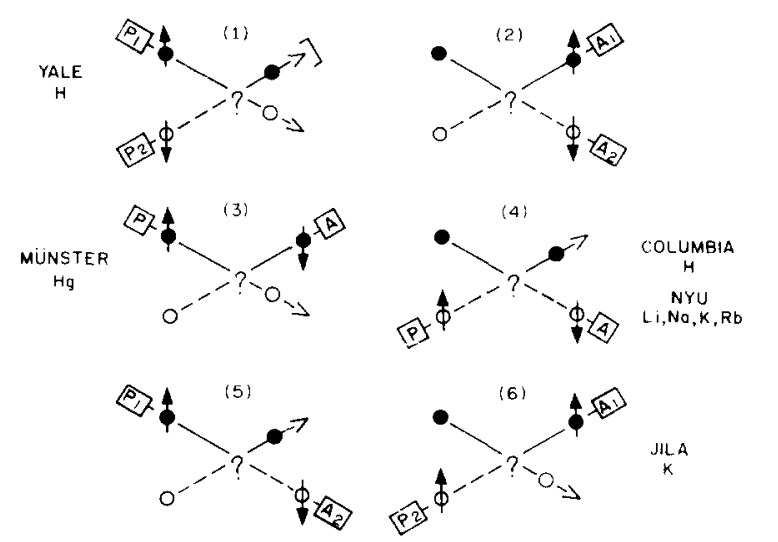

$\operatorname{JILA}$

FIG. I. Nonreactive scattering experiments with two polarization devices. Polarizers are denoted by $P$ and analyzers by $A$ in the appropriate boxes. Solid circles denote electrons while open circles denote atoms. The asymmetries which are measured by the experiments in a given row are specified by $A, A^{\prime}$, and $A^{\prime \prime}$ in accordance with Eqs. (4)-(12). Polarization experiments which have been carried out heretofore are identified by atomic species and the laboratory where they were performed: Yale $\mathrm{H}$-this work and Ref. 47; Münster $\mathrm{Hg}$-Ref. 53: Columbia $\mathrm{H}$-Ref. 48: NYU Li Na K Rb-Refs. 4-9; JILA K-Ref. 9.

measured. Specifically, the spin-averaged differential cross section,

$$
\frac{d \bar{\sigma}}{d \Omega}=1 / 4|f+g|^{2}+3 / 4|f-g|^{2},
$$

is determined. Only in the region of resonances can information be gleaned about the singlet and triplet amplitudes separately. Elsewhere, polarization experiments must be performed. Given the present limitations on polarized sources and polarization analyzers, these experiments must be limited to two polarization devices (sources or analyzers) each. Then in order to determine the three independent parameters, three experiments must be performed, two of which must be polarization experiments.

The six possible nonreactive experiments using two polarization devices are illustrated in Fig. 1. Only three of these provide independent information, with the two experiments in each row being redundant. The measurable quantities in each of these pairs of experiments can be determined from the cross-section summaries provided in Table 1.

Experiments (1) and (2) shown in Fig. 1 both result in the determination of the quantity $A$ given by

$$
A=\operatorname{Re}\left(f^{*} g\right)\left(\frac{d \bar{\sigma}}{d \Omega}\right)^{-1}
$$

In experiment (1), $A$ is given explicitly by the cross section asymmetry,

$$
A=\left(\sigma_{\uparrow}-\sigma_{\uparrow \uparrow}\right) /\left(\sigma_{\uparrow \downarrow}+\sigma_{\uparrow}\right)
$$

for the incident projectile electron and atomic electron spins antiparallel $(\uparrow \downarrow)$ and parallel $(\uparrow \uparrow)$. The experimentally measured asymmetry, $\Delta$, is related to $A$ by

$$
\Delta=P_{e} P_{a} A \text {. }
$$

where $P_{e}$ and $P_{a}$ are the degrees of polarization of the 
TABle I. Polarization processes ${ }^{\mathrm{a}}$ and cross sections.

\begin{tabular}{cl}
\hline \hline Process & Cross section \\
\hline$e \downarrow+A \uparrow \rightarrow e \downarrow+A \uparrow$ & $|f|^{2}$ \\
$e \downarrow+A \uparrow \rightarrow e \uparrow+A \downarrow$ & $|g|^{2}$ \\
$e \uparrow+A \uparrow \rightarrow e \uparrow+A \uparrow$ & $|f-g|^{2}$ \\
$e \uparrow+A \downarrow \rightarrow e \uparrow+A \downarrow$ & $|f|^{2}$ \\
$e \uparrow+A \downarrow \rightarrow e \downarrow+A \uparrow$ & $|g|^{2}$ \\
$e \downarrow+A \downarrow \rightarrow e \downarrow+A \downarrow$ & $|f-g|^{2}$ \\
\hline
\end{tabular}

a The electron is denoted by $e$, the atom by $A$, and the relative spin orientations by the arrows.

incident projectile and atomic electrons, respectively. In experiment (2), $A$ is again determined from Eq. (6), where $P_{e}$ and $P_{a}$ are now regarded as the analyzing powers of the electron and atom polarization detectors.

Experiments (3) and (4) both result in the determination of the quantity $A^{\prime}$ given by

$$
A^{\prime}=|g|^{2}\left(\frac{d \sigma}{d \Omega}\right)^{-1}-1,
$$

where $A^{\prime}$ is found from

$$
P_{e}^{\prime}=A^{\prime} P_{e}
$$

in experiment (3) and from

$$
P_{a}^{\prime}=A^{\prime} P_{a}
$$

in experiment (4), with $P_{e}^{\prime}$ and $P_{a}^{\prime}$ denoting the polarization of the scattered electrons and atoms, respectively. Experiments (5) and (6), on the other hand, both result in the determination of the quantity $A^{\prime \prime}$ given by

$$
A^{\prime \prime}=|f|^{2}\left(\frac{d \bar{\sigma}}{d \Omega}\right)^{-1}-1,
$$

where $A^{\prime \prime}$ is found from

$$
P_{a}^{\prime}=A^{\prime \prime} P_{e}
$$

in experiment (5), and from

$$
P_{e}^{\prime}=A^{\prime \prime} P_{a}
$$

in experiment (6). A comparison of Eqs. (3), (4), (7), and (10) reveals that the measurement of $d \bar{\sigma} / d \Omega$ along with two of the three quantities $A, A^{\prime}$, and $A^{\prime \prime}$ suffices to determine the three parameters needed to describe the scattering problem.

Until recently, all polarization experiments resulted in the determination of either $A^{4-8,48,53}$ or $A^{\prime \prime} .^{9}$ (In the case of $\mathrm{Hg}$ the effects of the spin-orbit interaction were taken into account in the analysis. ${ }^{53}$ ) With the development of the source whose application is described in this paper it has now become possible to determine the quantity $A$ through the performance of polarized electron-polarized atom experiments. The first experiments of this type, in which $\boldsymbol{A}$ was measured for elastic scattering and impact ionization in $e-\mathrm{H}$ collisions, have been reported elsewhere. ${ }^{47,59}$

In this paper we will describe the experimental details and principles of operation of the Fano-type polarized electron source which has been developed at Yale for electron-atom scattering. In Sec. I we will summarize the criteria which must be considered for polarized electron sources in general, and more specifically those which are important for crossed electronatom beams experiments. In Sec. II we will discuss the production of polarized electrons by means of the Fano effect, and we will describe the design and operation of the Yale source with emphasis on those characteristics which make it suitable for crossed beams studies. Finally in Sec. III we will summarize the operating characteristics of the source and speculate on some future polarized electron source developments.

\section{REQUIREMENTS OF POLARIZED ELECTRON SOURCES}

During the last fifteen years a wide variety of electron sources have been developed based upon a diversity of physical principles. ${ }^{26,27,56}$ Some of these sources, while interesting because of the underlying physics principles involved, are not at all practical for application to low-energy electron-atom scattering. Others, which originally showed promise for such application, have failed to live up to intensity and polarization expectations as useful laboratory instruments.

In selecting a source for a particular application it is desirable to base the selection on a set of criteria which provides an objective means of comparison of sources. The most frequently quoted criterion in polarized electron literature is the figure of merit, $\zeta$, given by

$$
\zeta=P_{e} \sqrt{I_{e}},
$$

where $I_{e}$ is the intensity of the source. However, this figure simply describes the statistical accuracy which can be achieved in an asymmetry measurement, independent of any external constraints. Of course each experiment imposes its own unique set of external constraints, and these constraints must be taken into account in the evaluations of the merits of any given source. Generally speaking, the salient characteristics of a source which impinge on the specific requirements of an experiment can be summarized as follows:

(1) Intensity, $I_{e}$.

(2) Polarization, $\boldsymbol{P}_{\mathrm{e}}$.

(3) Figure of merit, $\zeta$.

(4) Direction of polarization.

(5) Variation of intensity and beam phase space under polarization reversal.

(6) Maximum frequency of polarization reversal.

(7) Energy spread, $\Delta E$.

(8) Emittance, $\epsilon$.

(9) Stability.

(10) Percent time available.

(11) Mode of operation: dc or pulsed.

(12) Pulse length and repetition rate. 
In the absence of magnetic fields the emittance (characteristic 8 ) is defined by

$$
\epsilon=\rho \alpha,
$$

where $\rho$ is the radius of the beam at an image of the source, and $\alpha$ is the aperture angle, that is, half the apex angle of the cone which includes all the electron trajectories from one image point. Nonrelativistically, $\epsilon$ is inversely proportional to the square root of the beam energy, $E$. In the presence of a magnetic field, $H_{0}$, at the source, the emittance given by Eq. (14) is no longer an adequate description of the electron beam phase space. For the case of low-energy electron scattering, where the electron beam interacts with the atomic beam in a region of low magnetic field, typically $10^{-5}-10^{-7} \mathrm{~T}$, the emittance of the beam must be generalized to $^{60}$

$$
\epsilon^{*}=\rho_{0}\left(E_{0} / E\right)^{1 / 2}+(1 / 2)(e / m) \rho_{0}^{2} H_{0} / v
$$

where $\rho_{0}$ is the effective radius of the electron production region in the source, $E_{0}$ is the mean energy of the electrons at the time of production, $e / m$ is the electron charge to mass ratio, and $v$ is the final velocity of the electron beam at the interaction region, all in SI units.

The presence of a strong magnetic field in the source region not only can increase the emittance of the beam but also can produce significant changes in beam intensity and position under polarization reversal. These changes, which will ultimately limit the precision of an asymmetry measurement, occur because sources which employ strong magnetic fields usually require the reversal of the field to effect the reversal of the direction of the beam polarization, thereby changing the electron optical properties of the beam. For crossed beams electron-atom scattering experiments, small changes in the position of the electron beam can result in significant changes in beam overlap and hence cause appreciable systematic errors in the measured asymmetry. These systematic errors will be particularly evident if the scattered electron is observed by a detector which does not have uniform collection efficiency over the entire crossed beams interaction region.

A list of polarized electron sources which are either already operational, in the prototype stage, or in the proposed construction stage is given in Table II. As can be seen under the heading "Method of polarization reversal," all sources rely on one of four methods for reversing the direction of polarization: change in the angle of emission, change in the energy of emission, reversal of the source magnetic field, and reversal of the optical polarization of photoionizing or photoemitting radiation incident at the source. Of the four, the optical reversal method is least likely to result in variation of beam position and intensity under polarization reversal. With these variations of paramount importance for crossed-beams experiments, the choice of source is restricted to those relying upon the Fano effect, ${ }^{62,75-77}$ resonant two-photon ionization of $\mathrm{Cs},{ }^{65}$ optical pumping of a He discharge,${ }^{66}$ and photoemission from negative electron affinity GaAs. ${ }^{69,70.78}$ At the present time, the Fano effect sources have undergone the greatest development and are probably the best understood sources suitable for low-energy electron scattering, although the major development programs currently in progress on GaAs sources will probably make these sources most attractive for future work.

\section{PRODUCTION OF POLARIZED ELECTRONS}

\section{A. Background}

For heavy alkali atoms, the photoionization cross section as a function of wavelength passes through a deep but nonzero minimum. Seaton in $1951^{79}$ suggested that this nonzero minimum is a consequence of the spin-orbit coupling. An additional consequence, first suggested by Fano in $1969,{ }^{80}$ is that photoelectrons produced by circularly polarized light in this wavelength region are spin polarized.

The first direct experimental verification of spin-orbit perturbation of the continuum P-states of heavy alkali atoms was provided in 1969 by Lubell and Raith ${ }^{81}$ through the observation of the asymmetry in ioncounting rates for the photoionization of polarized $\mathrm{Cs}$ atoms by circularly polarized light. Shortly thereafter Kessler and co-workers ${ }^{82,83}$ observed the actual production of polarized electrons in the photoionization of unpolarized $\mathrm{Cs}$ by circularly polarized light. Extensive investigation of the spin-orbit coupling in continuum states of $\mathrm{K}, \mathrm{Rb}$, and $\mathrm{Cs}$ have been reported in detail by Baum et al. ${ }^{84,85}$

\section{B. Theory}

\section{1. "Fano effect"}

As a vehicle for describing the spin-orbit perturbation, Fano ${ }^{80}$ introduced the perturbation parameter $x$ which is a function of photon energy $E$ and is defined by

$$
x(E)=3 R^{0}(E) /[\Delta R(E)],
$$

with

$$
\begin{gathered}
3 R^{0}=2 R_{13}+R_{11} \\
\Delta R=R_{13}-R_{11},
\end{gathered}
$$

where $R^{0}$ is the radial matrix element for the dipole transition from the $2_{S_{1 / 2}}$ ground state to a final continuum $p$-state neglecting the spin-orbit interaction in the final state, and $R_{13}$ and $R_{11}$ are the perturbed radial matrix elements for the final $P$-state angular momenta $J^{\prime}=3 / 2$ and $1 / 2$, respectively. It can be shown ${ }^{80,85}$ that the polarization of photoelectrons produced in the photoionization of alkali atoms by circularly polarized light is given in terms of the perturbation parameter $x$ by

$$
P_{e}=\left[(2 x+1) /\left(x^{2}+2\right)\right] P_{p h},
$$


TABLE II. Characteristics of polarized electron sources.

\begin{tabular}{|c|c|c|c|c|c|c|c|c|c|c|}
\hline \multirow{2}{*}{\multicolumn{2}{|c|}{ Method }} & \multirow[b]{2}{*}{ Group } & \multirow{2}{*}{$\begin{array}{l}\text { Refer- } \\
\text { ence }\end{array}$} & \multirow[b]{2}{*}{ Status ${ }^{a}$} & \multicolumn{2}{|c|}{ Mode } & \multirow{2}{*}{$\begin{array}{c}\text { Pulse } \\
\text { length } \\
(\mu \mathrm{s})\end{array}$} & \multirow{2}{*}{$\begin{array}{c}\text { Peak } \\
\text { current } \\
\left(10^{9} \mathrm{e} / \text { pulse }\right)\end{array}$} & \multirow{2}{*}{$\begin{array}{l}\text { Rep } \\
\text { rate } \\
\text { (pps) }\end{array}$} & \multirow{2}{*}{$\begin{array}{c}\text { Average } \\
\text { current } \\
\text { (nA) }\end{array}$} \\
\hline & & & & & Pulsed & $\mathrm{dc}$ & & & & \\
\hline \multirow{2}{*}{\multicolumn{2}{|c|}{$\begin{array}{l}\text { Electron scattering from } \\
\text { unpolarized } \mathrm{Hg} \text { beam }\end{array}$}} & Münster & 53 & 0 & & $\begin{array}{l}X \\
X\end{array}$ & & & & $\begin{array}{l}0.1 \\
0.01\end{array}$ \\
\hline & & Stanford & 61 & 0 & & $X$ & & & & $10-35$ \\
\hline \multirow{5}{*}{ 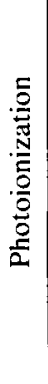 } & Fano effect $\mathrm{Rb}$ & Bonn & 62 & $\mathbf{D}$ & $X$ & & 0.012 & 2.2 & 50 & 18 \\
\hline & Fano effect Cs & Yale (This Work) & - & 0 & & $X$ & & & & 25 \\
\hline & $\begin{array}{l}\text { Polarized Li beam } \\
\text { "PEGGY" }\end{array}$ & $\begin{array}{l}\text { Yale-SLAC- } \\
\text { Bielefeld }\end{array}$ & 63 & 0 & $\begin{array}{l}X \\
X\end{array}$ & & $\begin{array}{l}1.6 \\
1.6\end{array}$ & $\begin{array}{l}2.6 \\
8.15\end{array}$ & $\begin{array}{l}180 \\
180\end{array}$ & $\begin{array}{r}72 \\
235\end{array}$ \\
\hline & $\begin{array}{l}\text { Optically pumped } \\
\text { polarized Li beam }\end{array}$ & $\begin{array}{l}\text { Yale-SLAC- } \\
\text { Bielefeld }\end{array}$ & 64 & 0 & $\mathrm{X}$ & & 1.6 & 20 & 180 & 576 \\
\hline & $\begin{array}{l}\text { Resonant two-photon } \\
\text { unpolarized Cs }\end{array}$ & FOM Amsterdam & 65 & 0 & & $\mathrm{X}$ & & & & $\sim 100$ \\
\hline \multicolumn{2}{|c|}{$\begin{array}{l}\text { Optically pumped } \mathrm{He} \\
\text { discharge }\end{array}$} & Rice & 66 & $\begin{array}{l}0 \\
0\end{array}$ & & $\begin{array}{l}X \\
X\end{array}$ & & & & $\begin{array}{c}100 \\
10^{4}\end{array}$ \\
\hline \multicolumn{2}{|c|}{ Field emission EuS } & Bielefeld & 67 & $\mathbf{D}$ & & $X$ & & & & 10 \\
\hline \multicolumn{2}{|c|}{ LEED $W$} & Rice & 68 & 0 & & $X$ & & & & 50 \\
\hline \multirow{6}{*}{ 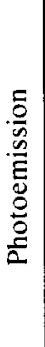 } & \multirow{5}{*}{ NEA GaAs } & ETH Zurich & 69 & 0 & & $\mathrm{X}$ & & & & $10^{3}$ \\
\hline & & NBS Gaithersburg & 70 & 0 & & $\mathrm{X}$ & & & & $10^{4}$ \\
\hline & & SLAC & 71 & $\mathbf{0}$ & $\mathrm{X}$ & & 1.6 & 100 & 180 & $3 \times 10^{3}$ \\
\hline & & Mainz & 72 & 0 & $\mathrm{X}$ & & 2 & 120 & 150 & $3 \times 10^{3}$ \\
\hline & & PMC Palaiseau & 73 & 0 & & $X$ & & & & $2 \times 10^{3}$ \\
\hline & $\mathrm{EuO}$ & ETH Zurich-SLAC & 74 & $\mathbf{0}$ & & $X$ & 1.2 & 3 & 6.7 & 3.2 \\
\hline
\end{tabular}

a Symbols used for source status are the following:

-Operational, O-Prototype, O-Proposed.

where $P_{p h}$ is the degree of circular polarization of the ionizing light. This functional dependence of $P_{e}$ on $x$, assuming $\boldsymbol{P}_{p h}=1$, is shown in Fig. 2. In Fig. 3 is illustrated the dependence of the perturbation parameter $x$ on the energy of the ionizing light for $\mathrm{Cs}, \mathrm{Rb}$, and K. ${ }^{85}$ From these two curves the polarization of photoelectrons, $P_{e}$, can be determined and is shown in Fig. 4 together with the measured polarization obtained in several experiments.

Since the first experimental verification of the production of spin-polarized electrons, several programs have been pursued to advance the state of Fano-effect polarized electron sources from the prototype to the operational. ${ }^{62,75-79}$ In the Yale program, Cs was chosen from among the heavy alkali atoms because it possessed the greatest bandwidth and largest cross section for the production of highly polarized electrons (see Appendix).

\section{Mott Scattering}

The polarization of electron beams is most frequently measured by means of Mott scattering, which is the relativistic elastic scattering of transversely polarized electrons by a heavy nuclear target. Because of the spin-orbit interaction involving the magnetic moment of the electron and the motional magnetic field of the target nucleus, electron scattering in the plane normal to the spin direction will show polarization-dependent effects which are, in general, functions of energy and scattering angle. These effects, first predicted by Mott in $1929^{86,87}$ and verified by Shull and co-workers in $1943,{ }^{88}$ are generally pronounced at high energies and large scattering angles. The analyzing power for Mott scattering is commonly called the Sherman function, $S$, after the detailed calculations published by Sherman in $1956,{ }^{89}$ and is defined by

$$
S(\theta)=i \frac{F(\theta) G^{*}(\theta)-F^{*}(\theta) G(\theta)}{|F(\theta)|^{2}+|G(\theta)|^{2}}
$$

where $F(\theta)$ is the spin-nonflip amplitude and $G(\theta)$ is the spin-flip amplitude resulting from the spin-orbit interaction. Many extensive studies of Mott scattering, both experimental ${ }^{90-93}$ and theoretical, ${ }^{94-98}$ have been carried out, making it the best understood process 


\begin{tabular}{|c|c|c|c|c|c|c|c|c|c|c|c|}
\hline \multirow{3}{*}{$\begin{array}{l}\text { Degree } \\
\text { of } \\
\text { polari- } \\
\text { zation }\end{array}$} & \multirow{2}{*}{\multicolumn{4}{|c|}{ Method of polarization reversal }} & \multirow{3}{*}{$\begin{array}{c}\text { Source } \\
\text { energy } \\
(\mathrm{eV})\end{array}$} & \multirow{3}{*}{$\begin{array}{c}\text { Energy } \\
\text { spread } \\
(\mathrm{eV})\end{array}$} & \multirow{3}{*}{$\begin{array}{c}\text { Magnetic } \\
\text { field } \\
\text { (G) }\end{array}$} & \multirow{3}{*}{$\begin{array}{l}\text { Emitlance } \\
\text { at source } \\
\text { energy } \\
(\text { mrad } \mathrm{cm})\end{array}$} & \multicolumn{3}{|c|}{ Brightness } \\
\hline & & & & & & & & & & & \\
\hline & Angle & Energy & Magnetic & Optical & & & & & Medium & High & high \\
\hline 0.22 & $X$ & $x$ & & & 80 & 1.0 & $\sim 0$ & - & & $x$ & \\
\hline 0.22 & $\mathrm{X}$ & $\mathrm{X}$ & & & 80 & 0.6 & $\sim 0$ & 一 & & $\mathrm{X}$ & \\
\hline $0.1-0.23$ & $\mathrm{X}$ & $\mathrm{X}$ & & & $50-180$ & - & -0 & 一 & & $\mathrm{x}$ & \\
\hline 0.65 & & & & $X$ & $120 \times 10^{3}$ & $<500$ & 120 & 1.0 & $\mathrm{X}$ & & \\
\hline 0.63 & & & & $X$ & 1000 & -3 & 0.05 & $<20$ & & $\mathrm{x}$ & \\
\hline 0.85 & & & $X$ & & $65 \times 10^{3}$ & 1500 & 200 & $<10$ & $X$ & & \\
\hline 0.35 & & & $X$ & & $65 \times 10^{3}$ & 1500 & 200 & $<10$ & $x$ & & \\
\hline 0.85 & & & & $X$ & $65 \times 10^{3}$ & 1500 & 200 & $<10$ & $X$ & & \\
\hline $0.3-0.6$ & & & & $X$ & - & 0.5 & $\sim 0$ & - & & $\mathrm{x}$ & \\
\hline 0.5 & & & & $\mathrm{X}$ & 500 & 0.5 & 5 & 5 & & $x$ & \\
\hline 0.3 & & & & $X$ & 500 & 0.5 & 5 & 5 & & $x$ & \\
\hline 0.85 & & & $X$ & & $2 \times 10^{3}$ & 0.1 & -50 & - & & & $X$ \\
\hline 0.37 & $X$ & $X$ & & & 80 & $<0.1$ & $\sim 0$ & - & & $x$ & \\
\hline 0.45 & & & & $\mathrm{X}$ & $<1$ & 0.2 & $\sim 0$ & 2 & & & $X$ \\
\hline 0.45 & & & & $x$ & $<1$ & 0.2 & $\sim 0$ & 2 & & & $X$ \\
\hline 0.45 & & & & $X$ & $70 \times 10^{3}$ & 0.2 & $\sim 0$ & $<10$ & & & $X$ \\
\hline 0.45 & & & & $X$ & - & 0.2 & -0 & - & & & $x$ \\
\hline-0.5 & & & & $\mathrm{X}$ & 5 & 0.2 & $\sim 0$ & - & & & $\mathrm{X}$ \\
\hline 0.6 & & & $X$ & & $\sim 2$ & $\sim 2$ & $21 \times 10^{3}$ & $1.5 \times 10^{-4}$ & $X$ & & \\
\hline
\end{tabular}

capable of measuring electron polarization. The theoretical calculations of Holzworth and Meister ${ }^{95}$ and $\mathrm{Lin}^{97}$ for $100-\mathrm{keV}$ scattering from $\mathrm{Au}$ are given in Fig. 5 together with the experimental measurements of Apalin et al. ${ }^{99}$ and Eckardt et al. ${ }^{100}$

\section{Apparatus}

\section{Polarized electron source.}

A scale drawing of the polarized electron source is presented in Fig. 6. The light source is a 1000-W highpressure $\mathrm{Hg}-\mathrm{Xe}$ arc lamp with Suprasil envelope. Light from the lamp, collected by an $f / 1.5$ quartz lens, passes through a cell $5 \mathrm{~cm}$ in length containing an aqueous solution of $\mathrm{NiSO}_{4}(100 \mathrm{~g} / \mathrm{l})$ which removes radiation of wavelength longer than $\sim 320 \mathrm{~nm}$. The entrance window of the cell is a Corning CS0-56 filter which removes radiation of wavelength shorter than $270 \mathrm{~nm}$. The exit window is a manually rotatable quartz plate coated externally with a dichroic linearpolarizing film. Since the macromolecules in the polarizing film are damaged after prolonged exposure to intense radiation, the bandwidth of the light is restricted by the $\mathrm{NiSO}_{4}$ and Corning filters prior to passage of the light through the polarizer. As an additional precaution, the polarizer is cooled by air and by circulation of the $\mathrm{NiSO}_{4}$ solution through a heat exchanger. This latter process also serves to prevent the $\mathrm{NiSO}_{4}$ solution from boiling.

After leaving the absorption cell, the linearly polarized uv light passes through a 300-nm zeroth-order quartz quarter-wave retardation plate (which has its optical axis oriented at $45^{\circ}$ with respect to the orientation of the linear polarizer) to produce circularly polarized light. A quartz lens then focuses the circularly polarized light into the ionization region estimated to be $3 \mathrm{~cm}$ long and $5 \mathrm{~mm}$ in diameter. The helicity of the light is reversed upon rotation of either the linear polarizer or quarter-wave plate by $90^{\circ}$.

The characteristics of the uv optics for the wavelength region of interest are given in Fig. 7. Also shown for comparison is the photoionization cross section for Cs. From Fig. 7(c) it can be seen that $\sim 120 \mathrm{~mW}$ of ionizing radiation of wavelength $250-318 \mathrm{~nm}$ is delivered to the ionization region.

In the ionization region, the focused, circularly 


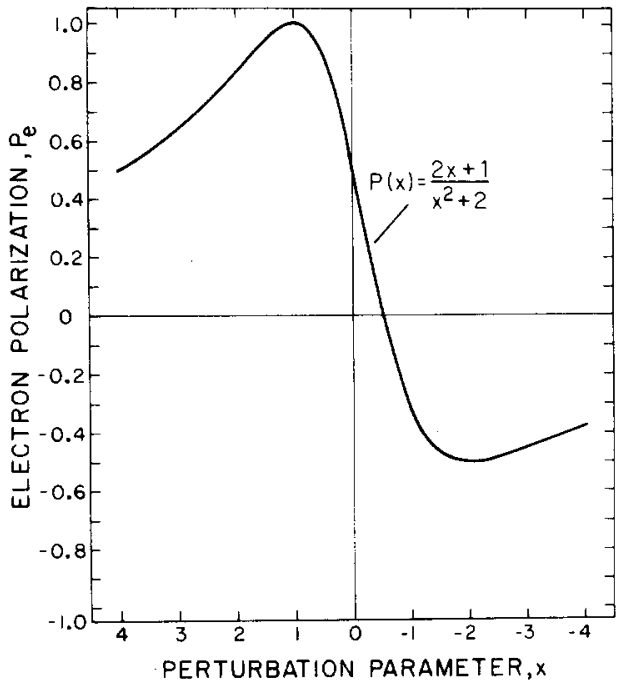

FIg. 2. Electron polarization, $P_{e}$, as a function of perturbation parameter, $x$, assuming $P_{p h}=1 .{ }^{85}$

polarized uv light intersects a beam of Cs atoms produced by the oven shown in Fig. 6 . The oven holds six 10-g glass ampoules of Cs metal and is designed to allow the ampoules to be broken one at a time while the system is under vacuum and at its operating temperature of $\sim 595 \mathrm{~K}$ at the lower chamber and $\sim 625 \mathrm{~K}$ at the upper chamber. The two-stage oven design is used to minimize the dimer $\left(\mathrm{Cs}_{2}\right)$ content of the beam. ${ }^{60}$ Directionality of the atomic beam is increased by a stainless steel multicapillary orifice structure, ${ }^{101,102} \sim 1.0 \mathrm{~mm}$ thick, containing $\sim 3 \times 10^{5} 14-\mu \mathrm{m}$-diam holes within an area of $\sim 1.5 \mathrm{~cm}^{2}$. The atomic flux, monitored by a surface ionization hot-wire detector ${ }^{103,104}$ located above the ionization region as shown in Fig. 6, is estimated to be $\sim 3 \times 10^{16}$ atoms $\mathrm{cm}^{-2} \mathrm{~s}^{-1}$ at the center of the ionization region. For an average beam velocity of

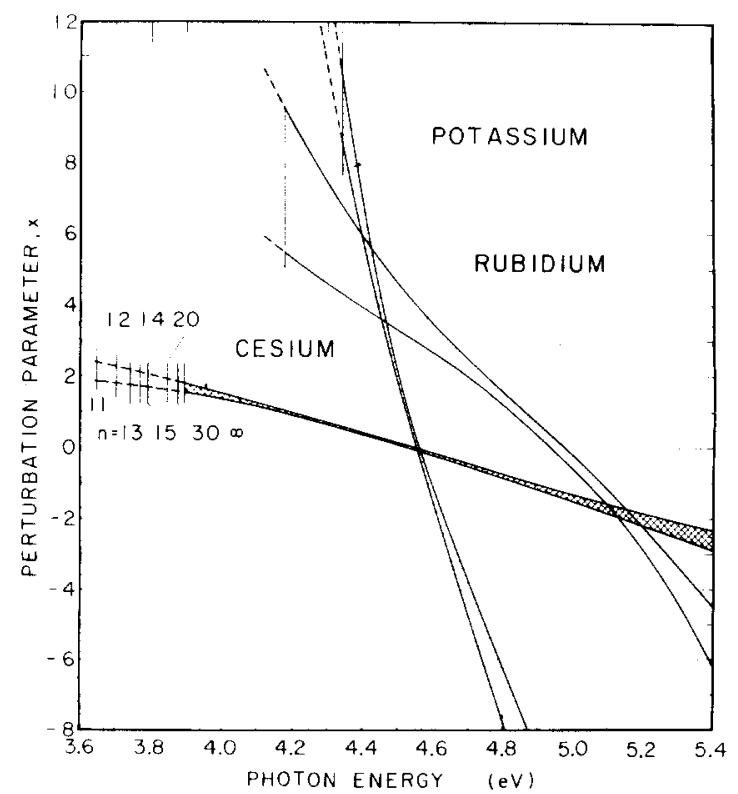

Fig. 3. Perturbation parameter, $x$, as a function of photon energy for potassium, rubidium, and cesium. ${ }^{\mathrm{RS}}$

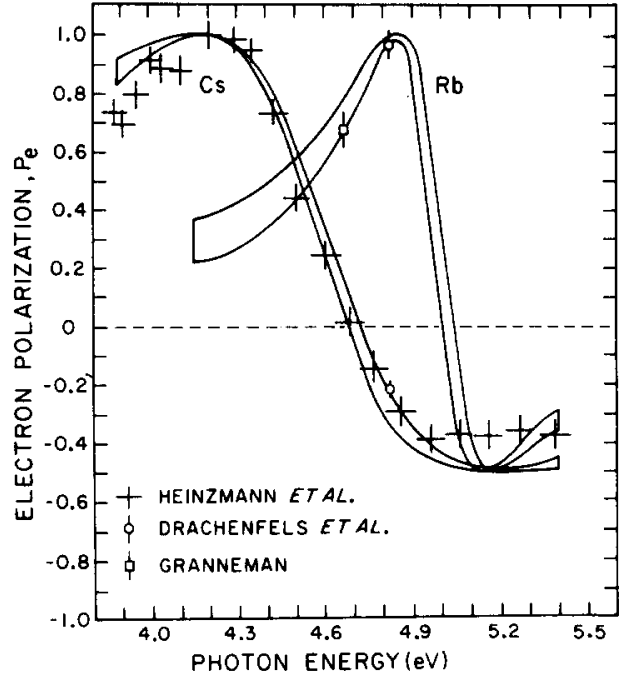

Fig. 4. Polarization parameter, $P_{\rho}$, as a function of photon energy for Rb and Cs. Curves are taken from results given by Baum et al. ${ }^{85}$ with bands representing one standard deviation uncertainties. Data points are from Heinzmann et al., ${ }^{76}$ Drachenfels et al., ${ }^{62}$ and Granneman. ${ }^{77}$

$\sim 3.2 \times 10^{4} \mathrm{~cm} / \mathrm{s}$ this flux corresponds to a density of $\sim 10^{12}$ atoms $/ \mathrm{cm}^{3}$ during operation. With $60 \mathrm{~g}$ of $\mathrm{Cs}$ in the oven, operation at this density can be maintained for $\sim 75 \mathrm{~h}$.

To facilitate transport, the longitudinally polarized electrons resulting from ionization of the Cs beam are accelerated to $1.0 \mathrm{keV}$ by maintaining the ionization region at $-1.0 \mathrm{kV}$ relative to ground. The oven and Freon-cooled vacuum chamber are also maintained at $-1.0 \mathrm{kV}$ in order to eliminate electrical discharges. The photoelectrons produced in the ionization region are extracted by a voltage gradient of $\sim 1 \mathrm{~V} / \mathrm{cm}$, which is produced by a pair of cylindrical electrodes as shown in Fig. 6. These electrodes are fabricated from stainless steel and are coated with colloidal graphite to minimize light reflection and photoemission from their surfaces. The results of computer calculations ${ }^{105}$ of the equipotential lines and electron trajectories are given in Fig. 8. A 200-mG axial field is established in the ionization region by the pair of Helmholtz coils

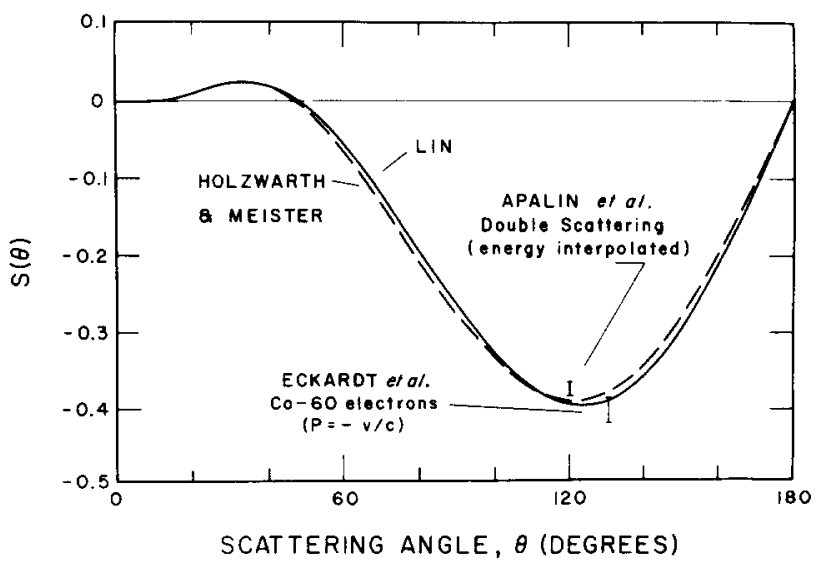

Fig. 5. Sherman function, $S(\theta)$, for scattering of $100-\mathrm{keV}$ electrons from screened Au nuclei, calculated by Lin $^{97}$ and Holzwarth and Meister ${ }^{95}$ with experimental data of Apalin et al. ${ }^{9 y}$ and Eckardt et al. ${ }^{100}$ 


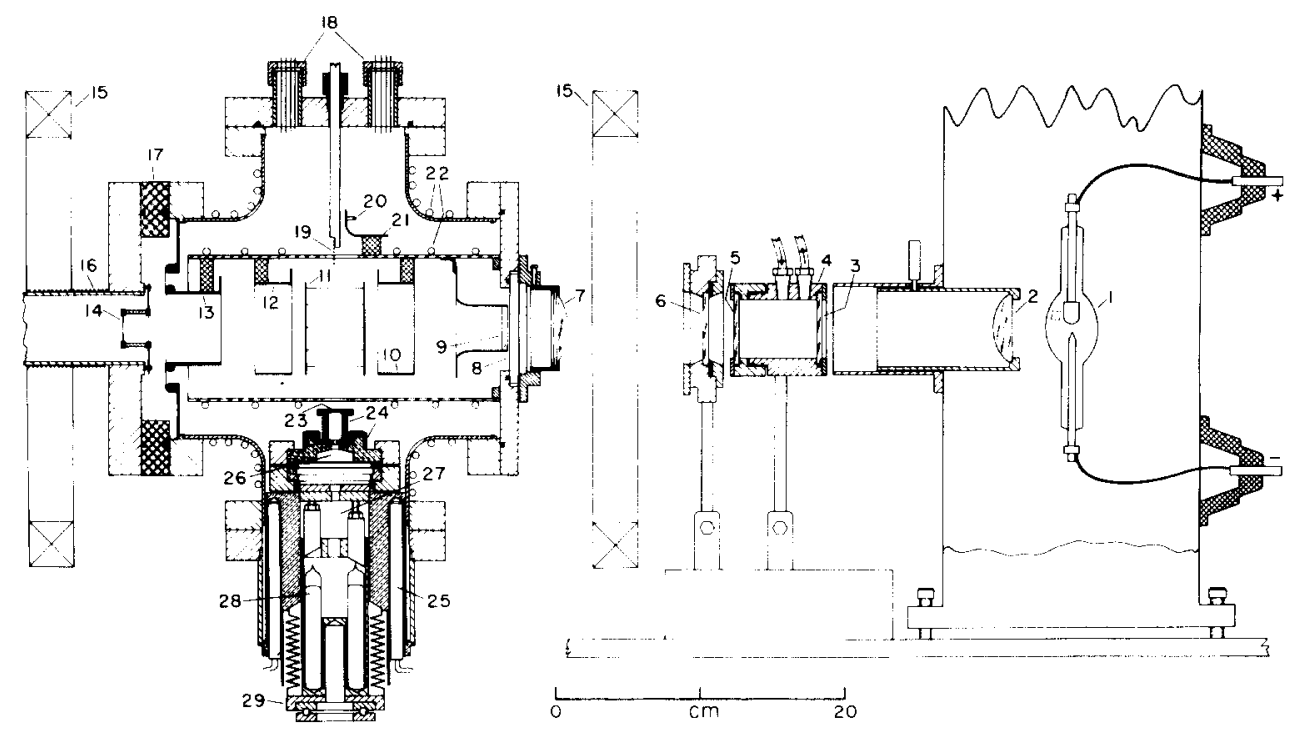

Fig. 6. Scale drawing of the Fano effect polarized electron source (side view). (1) - 1000-W Hg-Xe cw arc lamp with Suprasil envelope (Hanovia 977B-1); (2) $-f / 1.5$ quartz lens; (3) - Corning CS0-56 filter: (4) - $\mathrm{NiSO}_{4}$ absorption cell : (5) - dichroic linear polarizer (3M Company, 105 UV MRWR) on a $5-\mathrm{cm}$-diam quartz disk, rotatable: $(6)-3000-\AA$ zeroth-order quartz quarter-wave retardation plate, rotatable; (7)$f / 3.0$ quartz lens; (8) - Suprasi vacuum window; (9) - heated quartz disk to prevent $C$ s from fogging the window: $(10)$-repeller electrode: (11)-ionization region: (12)extractor electrode: (13)-focusing electrode; (14)-electron collimator: (15) - Helmholtz coils to establish a fixed $-200-\mathrm{mG}$ magnetic field in the ionization region collinear with the ionizing light: (16) - beam pipe with solenoid: (17)-Lucite insulating flange; (18)-electrical feedthroughs (Ceramaseal \#807B8177-1): (19)stainless steel mesh: (20) - hot-wire surface ionization Cs beam detector with (21) ion collector; (22) - Freon cooling pipes; (23) - stainless steel multicapilary orifice (Wintec Division, Brunswick Corp.): (24) - Thermocoax heating coils (North American Phillips): (25)-twelve 300-W heaters: (26) - oven upper chamber: (27) - oven lower chamber: (28) - six 10-g Pyrex ampoules of Cs metal (Kawecki Berylco Industries): (29)-bellows mechanism for breaking ampoules. All vacuum parts were machined from stainless steel unless otherwise noted. Oven temperatures were monitored by Chromel Alumel thermocouples (not shown). Also not shown is an externally operable Cs beam-blocking flag.

shown in Fig. 6. The direction of the electron polarization is collinear with the direction of the ionizing radiation, with the electron helicity the same as the light helicity.

For use in a low-energy crossed beams scattering experiment, the electrons are decelerated to the desired energy just prior to the interaction region. The energy spread of the electron beam, measured both by a decelerating filter lens ${ }^{106}$ and by the threshold behavior of hydrogen ion production, ${ }^{107}$ is $3.0 \mathrm{eV} \mathrm{FWHM}$, as shown in Fig. 9. For the purpose of initial electron optics tuning, a stainless steel mesh can be inserted into the ionization region at the image of the lamp to produce a $\sim 1 \mu \mathrm{A}$ beam of unpolarized "monoenergetic" $(\sim 0.2 \mathrm{eV}$ FWHM) electrons by photoemission from the mesh.

\section{Mott scattering analyzer}

A scale drawing of the polarization-analysis Mott scattering chamber is shown in Fig. 10. The 1.0-keV electrons from the Fano source are magnetically deflected by $45^{\circ}$ into the Mott branch of the experiment, thus preserving the longitudinal polarization, and are transported $\sim 1.5 \mathrm{~m}$ before entering the apparatus from the left in Fig. 10. The electrons are then accelerated to $7.0 \mathrm{keV}$ and spin rotated by $90^{\circ}$ in a Wien filter ${ }^{108}$ to produce the transversely polarized electrons needed for Mott scattering. After spin rotation, the electrons are accelerated to $-100 \mathrm{keV}$ and scattered from gold foil targets ranging in thickness from 27 to $53 \mu \mathrm{g} / \mathrm{cm}^{2}$, each having a Formvar backing $\sim 20 \mu \mathrm{g} / \mathrm{cm}^{2}$ thick.

A scale drawing of the Mott scattering region is shown in Fig. 11. The electrons scattered by $\pm 120^{\circ}$ in the plane normal to the incident polarization vector are detected by the pair of Si surface barrier detectors, as shown in
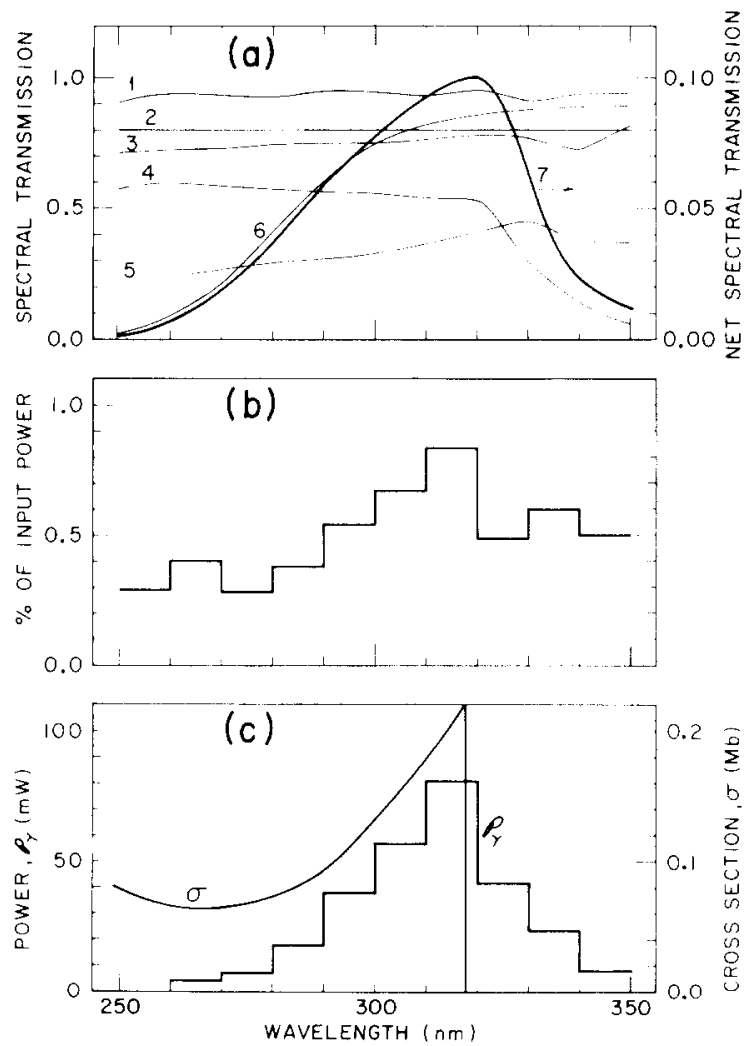

FIG. 7. Fano source uv optics characteristics. (a) The spectral transmission of the optical elements: (1) - Suprasil vacuum window: (2)-quartz lenses (estimate): (3)-quarter-wave plate; (4)- $\mathrm{NiSO}_{4}$ solution; (5)-linear polarizer (unpolarized incident light): (6) - Corning CS0-56 filter; (7) - Net spectral transmission. (b) The power output of the lamp in percent of input power $(1000 \mathrm{~W})$. The bar graph indicates the power produced within each $100-\mathrm{nm}$ wavelength segment (Hanovia specifications). (c) The photoionization cross section for cesium, $\sigma$, superimposed upon the power delivered to the ionization region, $P_{\gamma} . P_{\gamma}$ is the product of curve $(7)$ of $(a)$ and the lamp power given in (b), assuming $1000-W$ input power to the lamp. The ionization threshold for cesium is $318 \mathrm{~nm}$. 


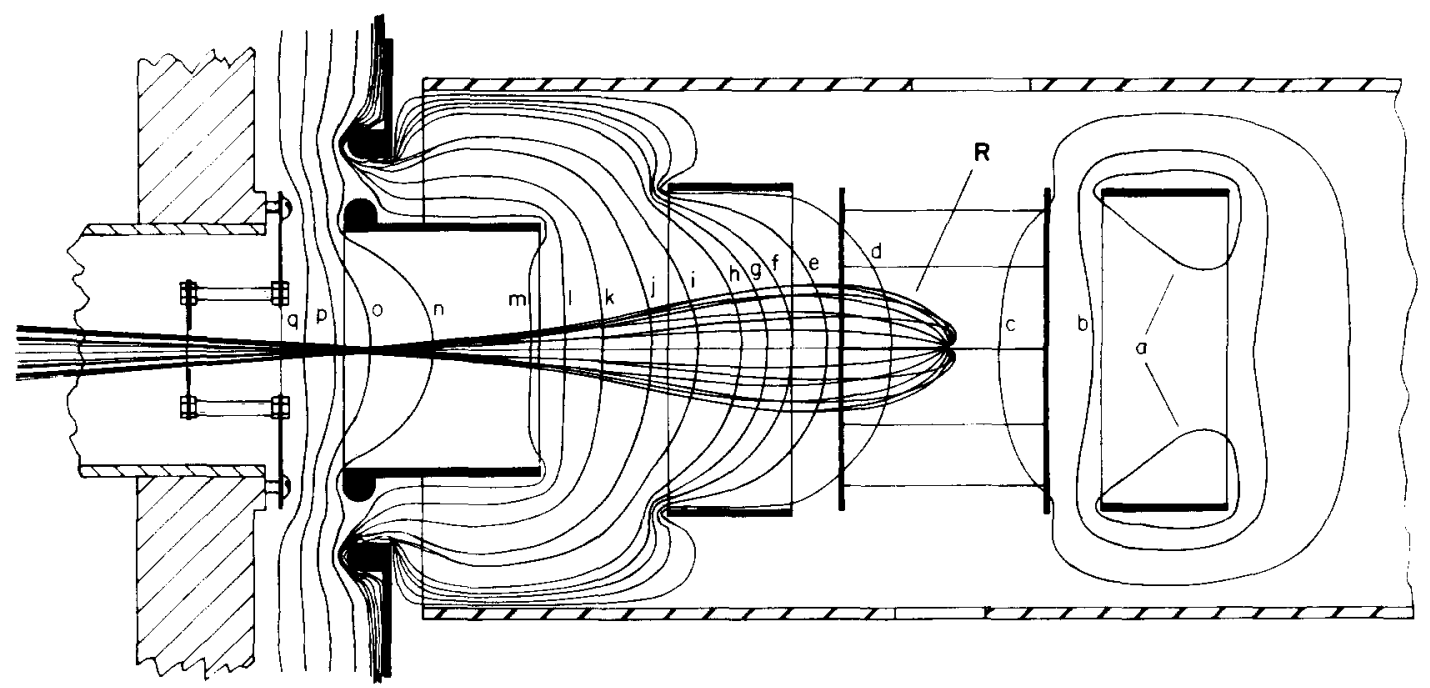

FIG. 8. Computer study of the Fano source ionization region. Equipotential lines are (in volts relative to ground) a, $-1005 ; b,-1003$; c, $-1001 ; \mathrm{d},-999 ; \mathrm{e},-997 ; \mathrm{f},-995 ; \mathrm{g},-993 ; \mathrm{h},-991 ; \mathrm{i},-980 ; \mathrm{j},-960 ; \mathrm{k},-940 ; 1,-920 ; \mathrm{m},-900 ; \mathrm{n},-800 ; \mathrm{o},-600 ; \mathrm{p},-400 ; \mathrm{q},-200$. Electron rays $(R)$ are calculated for electrons starting at the center of the ionization region with energy of $0.2 \mathrm{eV}$, and various angles in the plane of the figure. Other computer studies show that electrons produced on the surfaces of electrodes are not extracted from the Fano source. The longitudinal magnetic field in this simulation is $0.2 \mathrm{G}$.

Fig. 11. Since the Mott scattering apparatus is maintained at $\sim 100 \mathrm{kV}$ relative to ground, the amplified pulses from each detector are converted to analog optical signals using light emitting diodes. These optical signals are transmitted to ground potential through Lucite light pipes and reconverted to electrical signals by photomultipliers.

\section{Method of operation}

\section{Low-energy crossed-beams atomic collision experiment}

Because of the susceptibility of a low-energy atomic collision experiment to systematic errors associated with polarization reversal, as discussed in Sec. I B, the polarized electron source is designed and operated in a manner which minimizes these effects. In order to take advantage of the optical reversibility of a Fano effect source, the reversal of the electron polarization is automated by use of a stepping motor which rotates the quarter-wave plate at frequent intervals. (The linear polarizer is rotated manually at less frequent intervals.) The stepping motor is under the control of a PDP-15 computer, which also supervises the acquisition and storage of data.

Collision data are typically recorded in the following manner. The state-selected target atomic beam is chopped at $100 \mathrm{~Hz}$ for simultaneous background measurement, and the direction of target polarization is determined by a small $(\sim 100 \mathrm{mG})$ magnetic field in the scattering region colinear with the incident electron beam. With the target polarization and linear polarizer orientation held fixed, data are recorded in runs lasting 10-40 min, depending upon the counting rate of the particular experiment being performed. Within each run, data are collected by the computer in a manner indicated below. For each of four settings of the quarter-wave plate, $0,1,2$, and 3 , differing by $90^{\circ}$, counts are accumulated on a set of blind scalers for data intervals of typically $5-10 \mathrm{~s}$, the exact time being determined for normalization purposes by the charge collected in a Faraday cup located downstream from the scattering region. In addition to the event rate for the desired measurement, the computer also records the duration of the measurement, digitized integrated Faraday cup signal, and atomic beam composition informa-
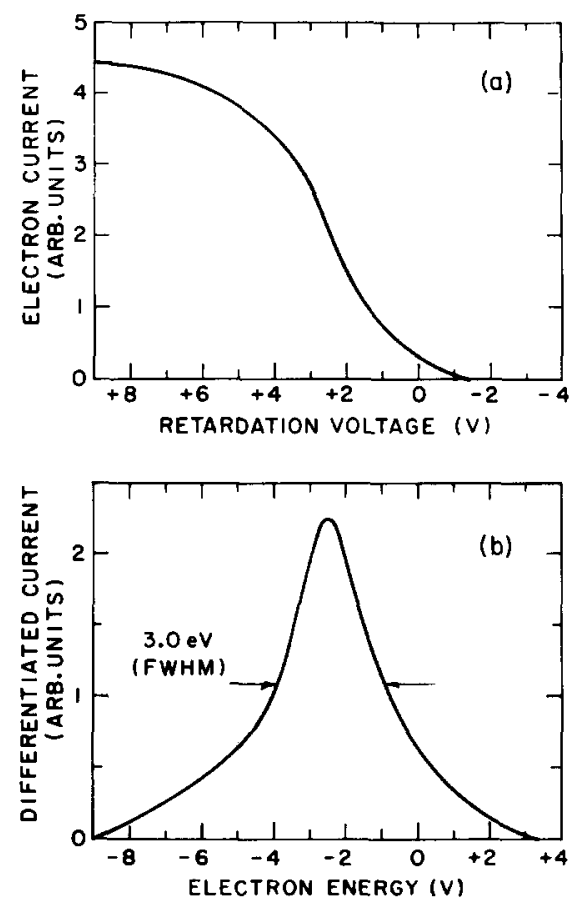

FIG. 9. Filter lens electron beam energy analysis. (a) Electron current transmitted by the lens as a function of retardation voltage, with voltage measured relative to the $-1 \mathrm{kV}$ Fano source supply. (b) Differentiated form of (a), indicating the electron energy spread (electron energy measured relative to $1 \mathrm{keV}$ ). Filter lens resolution is $0.4 \mathrm{~V}$ FWHM. 


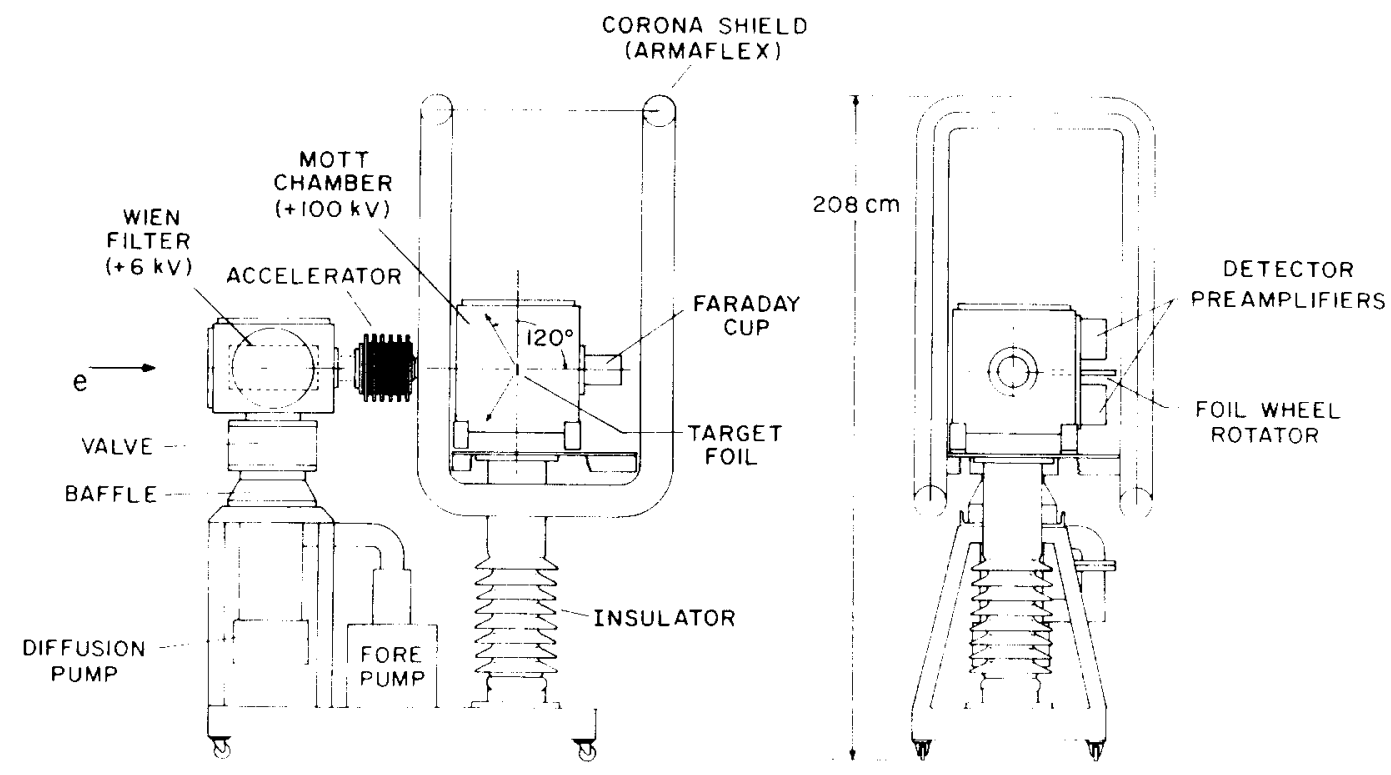

Fig. 10. Scale drawing of Mott scattering chambers. The Wien filter consists of crossed transverse electric and magnetic fields of $3.6 \mathrm{kV} / \mathrm{cm}$ and $44.3 \mathrm{G}$, respectively. With the Wien filter maintained at $+6 \mathrm{kV}$ with respect to ground, the beam energy in the Wien filter is $7 \mathrm{keV}$. The accelerator tube consists of 7 aluminum disks, connected by 1.7-G $\Omega$ resistors. The entire Mot1 scattering region, including detectors, preamplifiers, amplifiers, and detector bias supplies, is maintained at a potential of $+99 \mathrm{kV}$.

tion obtained from a mass analyzer monitor. Upon completion of a measurement for a given quarter-wave plate position, the blind scalers are recorded by the computer, the quarter-wave plate is rotated to effect electron polarization reversal, and data taking is resumed. At the end of the 10-40-min run the data are written onto magnetic tape, the linear polarizer is rotated manually, and a new run is started. After several such runs, the target polarization is reversed and the entire sequence of quarter-wave plate and linear polarizer rotations is repeated.

For each run, the real asymmetry, $\Delta_{R}$, is defined as

$$
\Delta_{R}=\frac{N_{+}-N_{-}-B_{+}+B_{-}}{N_{+}+N_{-}-B_{+}-B_{-}}
$$

where $N_{+(-)}$is the sum of scattered events for atomic beam-on and quarter-wave plate positions 0 and 2 ( 1 and 3 ), and $B_{+(-)}$is similarly defined for atomic beam-off events. In addition to the real asymmetry just defined, two false asymmetries, $\Delta_{1}$ and $\Delta_{2}$, can be constructed by taking different combinations of quarter wave plate positions in the numerator of Eq. (20), $0+1-2-3$ and $0+3-1-2$, respectively. For both the real and false asymmetries, the values of $\Delta$ measured for each run are combined according to their statistical weights to give averaged asymmetries. A check for systematic effects is accomplished by comparing the averaged false asymmetries, $\bar{\Delta}_{1}$ and $\bar{\Delta}_{2}$, with zero and by calculating the $\chi^{2}$ around zero for the sets of $\Delta_{1}$ and $\Delta_{2}$.

\section{Polarization measurements}

Mott scattering polarization measurements are interspersed between the runs at irregular intervals. A typical pulse-height spectrum of the signal from one of the two Si surface barrier detectors is shown in Fig. 12.
Discriminator thresholds are set as indicated by the arrow in the figure. Detected events occurring above threshold are gated according to the quarter-wave plate position at the Fano source, and are scaled on one of two pairs of scalers, one pair for positive helicity light and the other pair for negative helicity light. Counts are accumulated for 5-s intervals, with the quarter-wave plate rotated by $90^{\circ}$ between each interval. After an integer number of complete quarter-wave plate rotations, the scalers are recorded. This procedure is repeated for each of the four possible orientations of the linear polarizer. A complete polarization measurement consists of a total of $\sim 4 \times 10^{5}$ events, requires $\sim 4 \mathrm{~min}$, and results in a statistical uncertainty of $\sim 1.6 \times 10^{-3}$ in the measured Mott asymmetry.

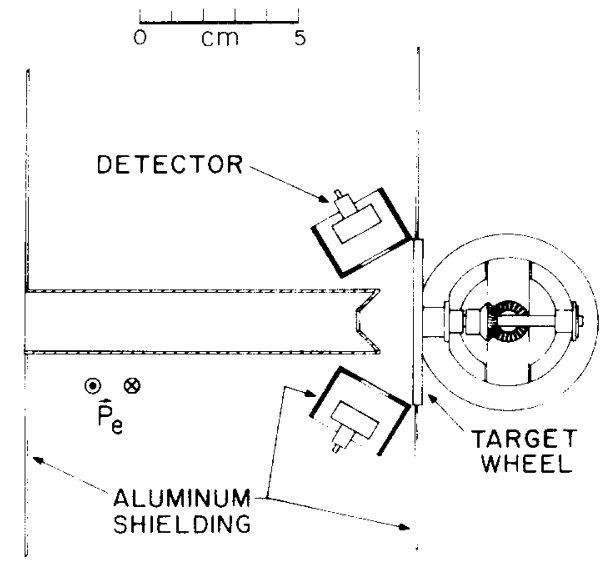

FIG. 11. Mott scattering region. The 100-keV transversely polarized electrons enter from the left and are scattered by one of six targets in the target wheel, which can be rotated while the system is under vacuum and at high voltage. The two surface barrier detectors are Ortec model SBEE100. Aluminum is used for shielding and chamber construction to maximize the energy loss of surfacescattered electrons which enter the detectors. 


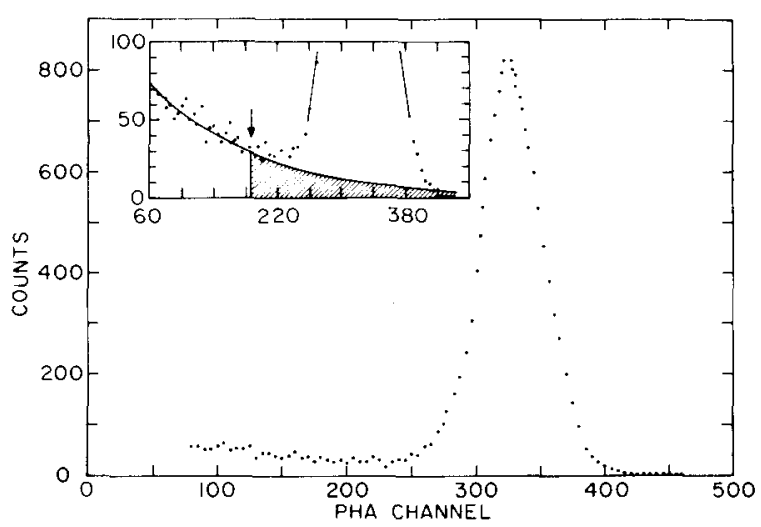

FIG. 12. Mott pulse-height analysis spectrum. Shaded area represents inelastic background subtraction. The arrow indicates the discriminator threshold.

\section{E. Mott data analysis}

Corrections to the Mott data must be made for the unpolarized inelastic background, for multiple and plural scattering, and for scattering from the Formvar backing. ${ }^{60}$ The inelastic background correction is accomplished by fitting the background counts to an exponential of the form $e^{-\beta n}$ as shown in Fig. 12, where $n$ is the channel number. The background counts, shown as the shaded area of the insert of Fig. 12, are typically found to be $\sim 6 \%$ of the total counts within the elastic peak.

The multiple and plural scattering correction is obtained from a series of polarization measurements made with different thickness target foils. Using an extrapolation to zero thickness of the form

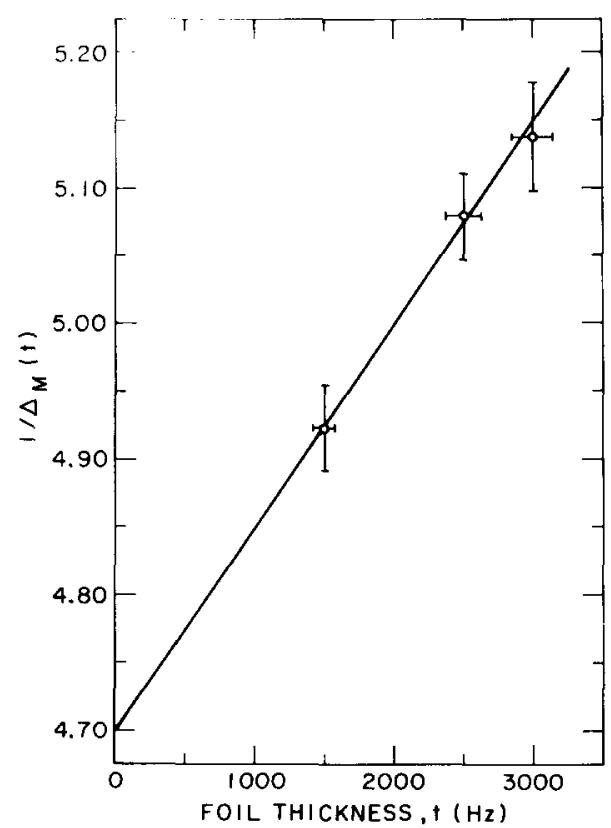

FIG. 13. Mott foil thickness extrapolation. The foil thickness in $\mathrm{Hz}$ was determined by piezo-electric frequency measurements during vacuum deposition of the foils. The foil thickness in $\mu \mathrm{g} / \mathrm{cm}^{2}$ can be obtained from the frequency measurement using the conversion factor $1 \mathrm{kHz}=17.7( \pm 20 \%) \mu \mathrm{g} / \mathrm{cm}^{2}$, which was determined by interferometric methods.

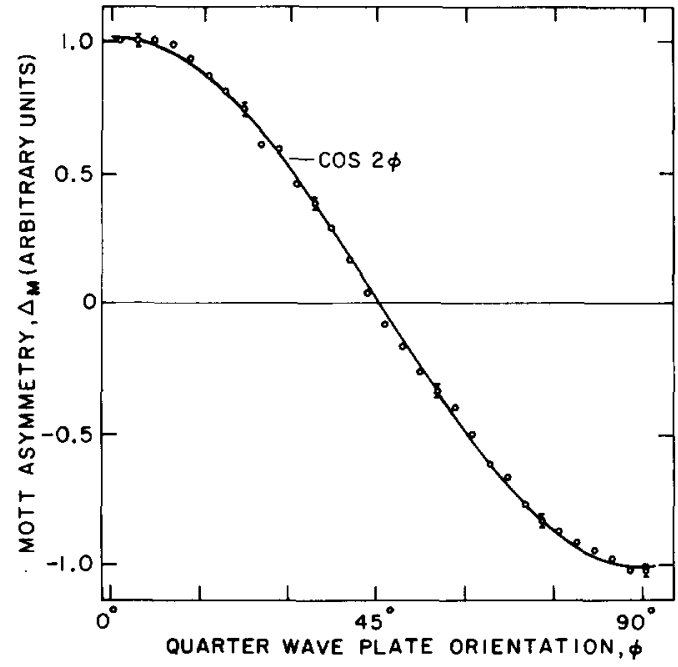

FIG. 14. Mott analysis asymmetry, $\Delta_{M}$, as a function of Fano source quarter-wave plate orientation, $\phi$.

$$
\frac{1}{\Delta_{M}(t)}=\frac{1}{\Delta_{M}(0)}(1+\alpha t)
$$

where $t$ is the foil thickness and $\alpha$ is a constant, the measured asymmetry corrected to zero thickness, $\Delta_{M}(0)$, is obtained, as illustrated in Fig. 13. Although the absolute thicknesses of the foils are only known to $\pm 20 \%$ from interferometric measurements, the relative thicknesses are known to $\pm 5 \%$ from piezoelectric measurements. Thus although $\alpha$ is determined with a precision of $22 \%, \Delta_{M}(0)$ can be determined with a relative precision of $1.7 \%$. [A value of $\alpha=(0.0018 \pm 0.0004) \mathrm{cm}^{2} /$ $\mu \mathrm{g}$ was measured, which agrees to within one standard deviation with previously reported measurements, assuming similar uncertainties in the absolute values of the foil thicknesses ${ }^{74,109}$ ] It should be noted that scattering from the Formvar backing was measured using a bare Formvar target, and was found to contribute less than $0.2 \%$ of the rate for the thinnest target $\left(27 \mu \mathrm{g} / \mathrm{cm}^{2}\right)$, within the elastic peak.

The measured Mott asymmetry, $\Delta_{M}$, is defined by

$$
\Delta_{M}=\frac{1-\xi}{1+\xi}
$$

where $\xi$ is given by

$$
\xi=\sqrt{\frac{N_{1}^{+}}{N_{2}^{+}} \cdot \frac{N_{2}^{-}}{N_{1}^{-}}} .
$$

Here $\mathrm{N}_{1}^{+}$and $\mathrm{N}_{2}^{+}$are the number of counts coming from detectors 1 and 2, respectively, for the case of positive helicity light, and $\mathrm{N}_{1}^{-}$and $\mathrm{N}_{2}^{-}$are the corresponding counts for negative helicity light. The quantities $N_{1}^{ \pm}$and $N_{2}^{ \pm}$contain corrections for inelastic background scattering and for detector and electronic noise. The Mott asymmetry is computed in this manner to offset any instrumental effect of differing effective detection efficiencies for the two detectors.

Once a value for $\Delta_{M}$ is established, the corresponding electron polarization, $P_{e}$, is calculated using the relation

$$
P_{e}=(1 / S) \Delta_{M}(0),
$$


where $S$ is the Sherman function. For the case of $120^{\circ}$ Mott scattering from $\mathrm{Au}$ at $100 \mathrm{keV}, S$ is known to be $0.391 \pm 0.008 .^{60,93,96}$

Several checks are made to insure the integrity of the measurement. First, the Wien filter spin rotator is scanned on both sides of its calculated operating point to insure that the spin rotation from longitudinal to transverse is $90^{\circ}$. In addition, the quarter-wave plate zero position is rotated by $90^{\circ}$ in steps of $3^{\circ}$ in order to ascertain that the relative orientation of the linear polarizer and quarter wave plate produces maximum polarization. The typical electron-polarization dependence upon quarter-wave plate orientation is shown in Fig. 14. Finally, the measured polarizations at each of the four Polaroid positions are compared for consistency. No deviations were found within statistical uncertainties.

\section{RESULTS AND CONCLUSIONS}

\section{A. Operating characteristics}

The operating characteristics of the Fano polarized electron source are summarized in Table III. The electron current was measured by a removable Faraday cup placed $-30 \mathrm{~cm}$ downstream from the exit of the electron source. Currents in excess of $25 \mathrm{nA}$ were produced, but more efficient operation was achieved with source intensities of $\sim 10 \mathrm{nA}$, resulting in a source lifetime of $\sim 75 \mathrm{~h}$ for an oven load of $60 \mathrm{~g}$ of Cs. The time required to reload the Cs oven was typically $24 \mathrm{~h}$.

The absolute electron energy, as measured by $\mathrm{H}^{+}$ production and by the decelerating filter lens, was found to depend upon Cs accumulation on the electrode surfaces, presumably due to the changes in the work function of those surfaces. A typical energy profile of the beam is shown in Fig. 9. The centrum of this profile was found to shift toward lower energy during the first few minutes of operation, but became stable after cesiation of the electrodes was complete. The typical energy shift was $\sim 1.5 \mathrm{~V}$.

The electron polarization was found to depend upon the extraction voltage gradient in the ionization region, as shown in Fig. 15. While this phenomenon was not studied in detail, it is thought that the degradation in electron polarization with decreasing extraction voltage may be attributable to $e-C$ s spin-exchange collisions. Indeed, assuming an atom density of $\sim 10^{12} \mathrm{~cm}^{-3}$, a

TABLE III. Polarized electron source characteristics.

\begin{tabular}{ll}
\hline Electron polarization, $P_{r}$ & $0.63 \pm 0.03$ \\
Electron current & $25 \mathrm{nA}$ \\
Maximum & $10 \mathrm{nA}$ \\
Average & $3.0 \mathrm{eV}$ \\
Energy spread (FWHM) & $20 \mathrm{mrad} \mathrm{cm}$ \\
Emittance at $1 \mathrm{keV}$ & $60 \mathrm{~g}$ \\
Oven capacity & $75 \mathrm{~h}$ \\
Oven lifetime (at $10 \mathrm{nA})$ & $10^{12} \mathrm{atoms} / \mathrm{cm}^{3}$ \\
Atomic beam density & Optical \\
Polarization reversal & \\
\hline \hline
\end{tabular}

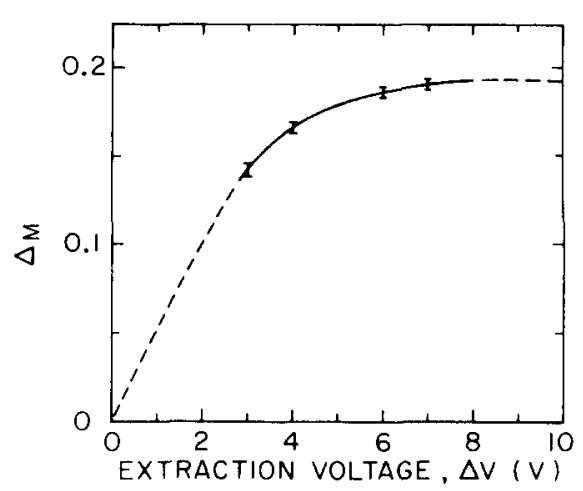

FIG. 15. Plot of the Mott asymmetry, $\Delta_{M}$, as a function of Fano source extraction voltage, $\Delta V$, between electrodes $(10)$ and (12) in Fig. 6.

spin-exchange cross section of $3.5 \times 10^{-14} \mathrm{~cm}^{2}$ (measured at thermal energies ${ }^{1}$ ), and an interaction path length of $\sim 10 \mathrm{~cm}$ (resulting from the helical motion of the electrons in the $200 \mathrm{mG}$ source field), a depolarization consistent with that shown in Fig. 15 for $\Delta V=1 \mathrm{~V}$ is obtained.

The decrease in electron polarization at lower extraction voltages necessitated a compromise between energy spread and polarization. Since maximum electron polarization was desired, the extraction voltage [the voltage between electrodes (10) and (12) in Fig. 6] was typically set at $7.0 \mathrm{~V}$, resulting in an energy spread of $\sim 3 \mathrm{eV}$ FWHM, as shown in Fig. 9. Under these conditions the electron polarization was measured to be $0.63 \pm 0.03$. As a consistency check on the electron polarization measurement, the circular polarization of the light, $P_{p h}$, was measured and the electron polarization averaged over all wavelengths, $\left\langle P_{p}\right\rangle$, was calculated according to

$$
\left\langle P_{e}\right\rangle=\frac{\int P_{p h}(\lambda) P_{e}{ }^{\text {ideal }}(\lambda) I_{p h}(\lambda) \sigma(\lambda) d \lambda}{\int I_{p h}(\lambda) \sigma(\lambda) d \lambda},
$$

where $P_{p}^{\text {ideal }}$ is the electron polarization for $P_{p h}=1$ from Fig. $4, I_{p h}(\lambda)$ is the spectral intensity, and $\sigma(\lambda)$ is the photoionization cross section, both from Fig. 7(c). With $P_{p h}(\lambda)=0.69 \pm 0.03,\left\langle P_{p}\right\rangle$ was determined to be $0.64 \pm 0.06$, consistent with the measured electron polarization.

Degradation of the dichroic linear polarizer due to energy absorption resulted in a slow decrease in electron polarization with time. This decrease was monitored during collision measurements and taken into account in the analysis of the collision data. Generally polarizers were replaced after approximately $100 \mathrm{~h}$ of usage, a time period which resulted in a decrease in electron polarization of $-30 \%$.

Two types of electron backgrounds were investigated: one corresponding to the Cs beam off and the other to the light beam off. The Cs beam-off background resulted from either photoemission from electrode surfaces or 
photoionization of residual Cs vapor. Since the polarization of the Cs beam-off background was measured to be identical within statistical uncertainties to that of the Cs beam-on contribution, and since the polarization of photoelectrons from bulk $\mathrm{Cs}$ is $<0.05,{ }^{110}$ it is concluded that essentially all of this background is the result of photoionization of the residual Cs vapor. Cs beam-off background currents were $\approx 10 \%$ of the total current.

Under normal operating source pressures of $\sim 3$ $\times 10^{-5}$ Torr, the light-off background was negligible. However, significant light-off background currents were observed under conditions of high chamber pressure $\left(\widetilde{10^{-4}}\right.$ Torr) and high Cs beam density. Under these conditions it was found that with the multicapillary orifice of the Cs oven biased as little as $-8 \mathrm{~V}$ with respect to the source electrodes, a glow discharge could be maintained resulting in copious numbers of unpolarized electrons.

The stability of the electron beam under polarization reversal was dramatically demonstrated by measurements of the false asymmetries for electron impact ionization of atomic hydrogen, ${ }^{59}$ where $\bar{\Delta}_{1}$ and $\bar{\Delta}_{2}$ were found to be consistent with zero at a level of $4 \times 10^{-4}$, a value limited only by the statistical accuracy of the measurements. Potentially, however, differential electron scattering measurements provide an even more sensitive test of systematic uncertainties associated with polarization reversal, since a differential scattering electron detector views a small invariant region generally constituting only a fraction of the crossed beams overlap volume. This situation is in sharp contrast to that of the impact ionization ion detector which viewed a region somewhat larger than the full beam overlap volume. From $90^{\circ}$ elastic scattering measurements it was found, nonetheless, that $\bar{\Delta}_{1}$ and $\bar{\Delta}_{2}$ were still consistent with zero at the statistical level of accuracy of $2 \times 10^{-3}$.

\section{B. Alternative sources for future measurements}

Although many polarized electron sources have been studied during the last 15 years, to date no other method of polarized electron production has been developed that is as suitable for use in low-energy crossed-beams atomic collision experiments as a Fano effect source. In fact, few of the prototype or proposed sources summarized in Table II are appropriate for lowenergy collision work because they do not meet the requirements of small energy spread and high brightness, and the desirability of optical reversability. It is clear, however, that the incipient development of an operational high-intensity GaAs source could greatly expand the range of possible low-energy polarized electron-atom collision experiments. In addition, if either the systematic effects associated with polarization reversal can be sufficiently suppressed or if the atomic polarization can be rapidly reversed (by optical pumping, for example), then field emission sources might prove very useful by virtue of their inherently narrow energy spread, very small emittance, and high polarization.

\section{ACKNOWLEDGMENTS}

We wish to thank Professor V. W. Hughes and W. Raith for their encouragement, support, and advice during the many years of development of the techniques described in this paper. We also wish to thank Dr. J. Ladish for his contributions during the early phases of the experimental program. In addition, we wish to express to L. Trudell, D. Constantino, J. Brosious, A. Disco, and the J. W. Gibbs machine shop our appreciation for their vital technical assistance. Finally, we wish to acknowledge the untiring help of Pat Fleming and Laurie Liptak in the preparation of this manuscript.

This research supported in part by the National Science Foundation under Grant No. PHY76-84469 and the U. S. Office of Naval Research under Contract No. NOOO14-76-C-0077.

\section{APPENDIX}

In this section we consider the possible advantages that might accrue through the use of a laser as the light source for photoionization. It is possible that with sufficient redesign and experimentation, the source might be successfully modified to take advantage of the smaller image size and higher brightness of a laser beam. Such modifications might well ultimately result in a polarized electron beam with a narrower energy spread and higher intensity, but these projections would be very speculative at best.

Rather than speculate on the future we will simply present an analysis of the advantages and disadvantages of incorporating a laser into the present source. It is clear that since a laser produces $100 \%$ linearly polarized, monochromatic light, it is possible to obtain $100 \%$ circularly polarized light. Then with the wavelength chosen at or near the peak of the polarization curve given in Fig. 4, nearly $100 \%$ electron polarization can be obtained. Moreover, the narrow beam diameter associated with a laser permits the use of a Pockels cell ${ }^{111}$ quarter-wave retarder, rather than a conventional quartz retardation plate, thereby permitting the photon helicity to be reversed more precisely and more rapidly. As a consequence, the systematic effects associated with polarization reversal, in principle, can be made vanishingly small.

An additional advantage of the smaller laser beam image is the immediate reduction in the emittance of the source by approximately one order of magnitude, a reduction which, for extremely low-energy electron scattering experiments, can be of paramount importance. Finally, the smaller image reduces the probability of unpolarized background electrons produced by photoemission from electrode surfaces, a process which, although a minor contribution under operation in the present source, did depend rather critically upon optics 
TABLE AI. Comparison of Fano source performance for various light sources.

\begin{tabular}{|c|c|c|c|c|}
\hline Characteristic & Arc lamp & Dye laser & \multicolumn{2}{|c|}{ Argon ion laser } \\
\hline $\begin{array}{l}\text { Fundamental } \\
\text { wavelength }\end{array}$ & $270-320 \mathrm{~nm}$ & $596 \mathrm{~nm}$ & $514.5 \mathrm{n}$ & \\
\hline $\begin{array}{l}\text { Fundamental } \\
\text { power }\end{array}$ & $120 \mathrm{~mW}$ & $2 W$ & $\begin{array}{l}15 W(a \\
40 W(a\end{array}$ & $\begin{array}{l}14.5 \mathrm{~nm}) \\
\text { ines) }\end{array}$ \\
\hline SHG crystal & & $\operatorname{KDA}\left(40^{\circ} \mathrm{C}\right)$ & ADP & $\left.0^{\circ} \mathrm{C}\right)$ \\
\hline $\begin{array}{l}\text { SHG wave- } \\
\text { length }\end{array}$ & & $298 \mathrm{~nm}$ & 257.25 & \\
\hline SHG power & & $1.5 \mathrm{~mW}$ & $379 \mathrm{~mW}$ & \\
\hline $\begin{array}{l}\text { Alkali atom } \\
\text { Electron } \\
\text { polariza- } \\
\text { tion, } P \text {. } \\
\text { Relative figure } \\
\text { of merit, } \zeta\end{array}$ & 0.63 & 0.11 & 0.97 & 0.28 \\
\hline
\end{tabular}

adjustments. The monochromaticity of the laser light, on the other hand, makes possible the use of antireflecting coatings on all optical elements, thereby improving the overall optical efficiency of the system.

While many of these advantages are difficult to quantify, the relative figures of merit, $\zeta$, for potential use of laser systems, as opposed to the arc lamp system currently used, can be evaluated rather easily. For the broadband light from the arc lamp, the figure of merit must be averaged over wavelength with the consequence that Eq. (13) becomes

$\zeta=\left\langle P_{e}\right\rangle \sqrt{\left\langle I_{e}\right\rangle}=\frac{\int P_{p h}(\lambda) P_{e}{ }^{\text {ideal }}(\lambda) I_{p h}(\lambda) \sigma(\lambda) d \lambda}{\sqrt{\int I_{p h}(\lambda) \sigma(\lambda) d \lambda}}$.

For monochromatic light of wavelength $\lambda_{0}$ Eq. (Al) reduces to

$$
\zeta=P_{p h}\left(\lambda_{0}\right) P_{e}^{\text {ideal }}\left(\lambda_{0}\right) \sqrt{I_{p h}\left(\lambda_{0}\right) \sigma\left(\lambda_{0}\right)}
$$

Table AI shows the results of the calculations for $\zeta$ for several choices of light sources. Both $\mathrm{Rb}$ and $\mathrm{Cs}$ have been considered as candidate alkali atoms, with the other alkalis being rejected for either reasons of low intensity or low polarization or both. For ease of comparison, the electron polarization, $P_{e}{ }^{i d e a t}$, and the photoionization cross section, $\sigma$, are shown together as functions of wavelength for $\mathrm{Cs}$ and $\mathrm{Rb}$ in Fig. Al. Since the wavelengths of interest are below $300 \mathrm{~nm}$, second harmonic generation (SHG) has been employed with the laser sources. ${ }^{113-116}$ We have restricted ourselves to $\mathrm{cw}$ lasers, although for some applications (time-of-flight experiments, for example) pulsed lasers might be advantageous. Indeed, pulsed lasers would give SHG peak powers several orders of magnitude higher than the $\mathrm{cw}$ average powers listed. The lasers listed in Table AI are commercially available from several

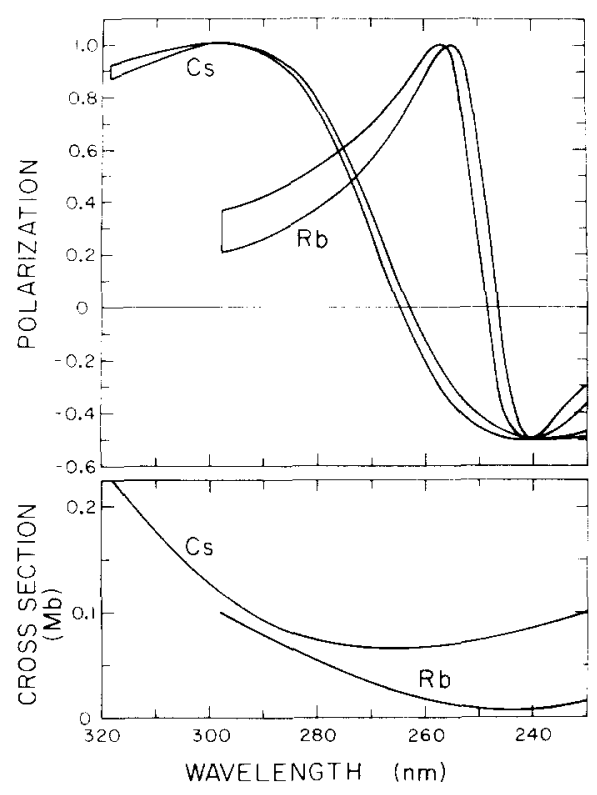

FIg. A 1. Photoelectron polarization, $P_{p m}{ }^{\text {ietert }}$, above ${ }^{85}$ and photoionization cross section, $\sigma$, below ${ }^{112}$ for $\mathrm{Cs}$ and $\mathrm{Rb}$, as functions of wavelength.

companies. It has been assumed that the dye laser is pumped by a $9-\mathrm{W}$ (all lines) argon ion laser.

From the results given in Table AI it can be seen that the simple incorporation of a commercially available laser system into the existing source does not result in improved performance when judged only by the figure of merit, $\zeta$. On the other hand, the possible advantages of smaller emittance might make the incorporation of a laser system scientifically worthwhile.

a' Present address: Bell Telephone Laboratories, Holmdel, NJ 07733

b) Present address: University of Bielefeld, Fakultät für Physik. Bielefeld, West Germany.

${ }^{1}$ L. C. Balling, R. J. Hanson, and F. M. Pipkin. Phys. Rev. 133, A607 (1964).

2 L. C. Balling and F. M. Pipkin, Phys. Rev. 136, A46 (1964).

${ }^{3}$ L. C. Balling, Phys. Rev. 151, 1 (1966).

K. Rubin, B. Bederson. M. Goldstein, and R. E. Collins, Phys. Rev. 182, 201 (1969).

s R. E. Collins, B. Bederson, and M. Goldstein, Phys. Rev. A 3, $1976(1971)$

'M. Goldstein, A. Kasdan, and B. Bederson. Phys. Rev. A 5, 660 (1972).

- B. Bederson and T. M. Miller, in Electron and Photon Interactions with Atoms, edited by $\mathrm{H}$. Kleinpoppen and M. R. C. McDowell (Plenum, New York, 1976), pp. 191-202.

${ }^{*}$ B. Jaduszliwer, N. D. Bhaskar, and B. Bederson, Phys. Rev. A 14, 162 (1976).

9 D. Hils, M. V. McCusker, H. Kleinpoppen, and S. J. Smith, Phys. Rev. Lett. 29, 398 (1972).

${ }^{10}$ D. M. Campbell, H. M. Brash, and P. S. Farago. Phys. Lett 36A, 449 (1971).

11 P. M. Stone and J. R. Reitz, Phys. Rev. 131, 2101 (1963).

12 W. R. Garrett and R. A. Mann, Phys. Rev. 130, 658 (1963)

13 W. R. Garrett and R. A. Mann, Phys. Rev. 135, A580 (1964).

14 W. R. Garrett, Phys. Rev. 140, A705 (1965)

1.5 P. G. Burke and A. J. Taylor, J. Phys. B 2, 869 (1969)

${ }_{16}$ D. W. Norcross, J. Phys. B 4, 1458 (1971).

${ }_{17}$ E. Karule, J. Phys, B 5, 2051 ( 1972).

${ }^{18}$ D. L. Moores and D. W. Norcross, J. Phys. B 5, 1482 (1972)

${ }^{19}$ G. D. Carse and D. W. Walker, J. Phys. B 6, 2529 (1973).

${ }^{20}$ P. G. Burke and J. F. B. Mitchell, J. Phys. B 7, 214 (1974).

${ }^{21}$ D. L. Moores, J. Phys. B 9, 1329 (1976).

${ }^{22}$ D. W. Walker, Adv. Phys. 20, 257 (1971)

${ }^{23}$ D. W. Walker, in Electron and Photon Interactions with Atoms, edited by $\mathrm{H}$. Kleinpoppen and M. R. C. McDowell (Plenum.

New York, 1976), pp. 203-213. 
24 J. Kessler, Rev. Mod. Phys. 41, 3 (1969).

${ }^{25}$ W. Raith in Atomic Physics, edited by V. W. Hughes, B. Bederson, V. W. Cohen, and F. M. J. Pichanick (Plenum, New York. 1969), pp. 389-415.

${ }^{26}$ J. Kessler, Polarized Electrons (Springer Verlag, Berlin, 1976).

${ }_{27}$ M. S. Lubell, in Atomic Physics 5 , edited by R. Marrus, M. Prior, and H. Shugart (Plenum, New York, 1977), pp. $325-373$.

${ }^{28}$ W. Franzen and R. Gupta, Phys. Rev. Lett. 15, 819 (1965)

${ }^{29}$ E. Reichert and H. Deichsel, Phys. Lett. 25A, 560 (1967).

30 T. Heindorff, J. Hofft, and E. Reichert, J. Phys. B 6, 477 (1973).

31 P. G. Burke and K. Smith, Rev. Mod. Phys. 34, 458 (1962).

32 P. G. Burke, D. F. Gallaher, and S. Geltman, J. Phys. B 2, 1142 (1969).

${ }^{33}$ P. G. Burke, in Atomic Physics, edited by V. W. Hughes, B. Bederson, V. W. Cohen, and F. M. J. Pichanick (Plenum, New York, 1969), pp. 265-294.

${ }^{34}$ R. J. Drachman and A. Temkin, in Case Studies in Atomic Collision Physics $I$, edited by $E$. W. McDaniel and M. R. C. McDowell (North Holland, Amsterdam, 1972), pp. 399-481.

${ }^{35}$ J. Callaway and J. F. Williams, Phys. Rev. A 12, 2312 (1975).

${ }^{36}$ See, for example S. Geltman. Topics in Atomic Collision Theory (Academic, New York, 1969), pp. 50-66, 115-131.

37 A. Temkin and J. C. Lamkin, Phys. Rev. 121, 788 (1961).

${ }^{38}$ C. Schwartz, Phys. Rev. 124, 1468 (1961).

${ }^{39}$ P. G. Burke and H. M. Schey, Phys. Rev. 126, 163 (1962)

40 M. K. Gailitis, in Proceedings of the Fourth International Conference on the Physics of Electronic and Atomic Collisions, Quebec, 1965 , edited by L. Kerwin and W. Fite (Science Bookcrafters, Hastings-on-Hudson, NY, 1965), pp. 10-14.

41 R. L. Armstead, Phys. Rev. 171, 91 (1968).

$42 \mathrm{~J}$. F. Williams, in Electron and Photon Interactions with Atoms, edited by H. Kleinpoppen and M. R. C. McDowell (Plenum, New York, 1976), pp. 309-338

${ }_{43}$ B. H. Bransden, J. P. Coleman, and J. Sullivan, J. Phys. B 5, 546 (1972)

44 J. C. Y. Chen, L. Hambro, A. L. Sinfailam, and K. T. Chung, Phys. Rev. A 7, 2003 (1973).

${ }^{45}$ G. Khayrallah, Phys. Rev. A 14, 2064 (1976).

${ }^{46} \mathrm{~F}$. W. Byron and C. J. Joachain, J. Phys. B 10, 207 (1977).

${ }^{47}$ M. J. Alguard, V. W. Hughes, M. S. Lubell, and P. F Wainwright, Tenth International Conference on the Physics of Electronic and Atomic Collisions, Paris, July 21-27, 1977 Abstracts of Papers (Commisariat a L'energie Atomique-Paris, 1977), pp. 506-507.

${ }^{48}$ W. Lichten and S. Schultz, Phys. Rev. 116, 1132 (1959),

${ }^{49}$ H. Kleinpoppen, Phys. Rev. A 3, 2015 (1971).

50 K. Blum and H. Kleinpoppen, Phys. Rev. A 9, 1902 (1974).

"1 P. S. Farago, J. Phys. B 7, L28 (1974)

${ }^{2} \mathrm{~K}$. Blum and H. Kleinpoppen, in International Journal of Quantum Chemistry Symposium No. 9, edited by P. Löwdin (Wiley, New York, 1975), pp. 415-424

G. F. Hanne and J. Kessler, J. Phys. B 9, 791 (1976)

${ }^{34}$ G. F. Hanne, J. Phys. B 9, 805 (1976)

is B. L. Moiseiwitsch. J. Phys. B 9, L245 (1976)

56 J. Kessler, in Atomic Physics 3 , edited by S. J. Smith and G. K. Walters (Plenum, New York, 1973), pp. 523-542

$\Rightarrow$ B. Bederson, in Atomic Physics 3, edited by S. J. Smith and G. K. Walters (Plenum, New York, 1973), pp. 401-427.

${ }^{*} \mathrm{E}$. Reichert, in Electron and Photon Interactions with Atoms, edited by $\mathrm{H}$. Kleinpoppen and M. R. C. McDowell (Plenum, New York, 1976), pp. 215-228

59 M. J. Alguard, V. W. Hughes, M. S. Lubell, and P. F Wainwright, Phys. Rev. Lett. 39, 334 (1977)

6) V. W. Hughes, R. L. Long, Jr., M. S. Lubell, M. Posner and W. Raith, Phys. Rev. A 5, 195 (1972).

6. W. A. Bonner, M. A. Van Dort, and M. R. Yearian. Nature 258, 419 (1975)

${ }^{62}$ W. von Drachenfels, U. T. Koch. Th. M. Müller, W. Paul, and $H . R$. Schaefer. Nucl Instrum Methods 140, 47 (1977)

6:3 M. J. Alguard, G. Baum, J. Clendenin, V. W. Hughes, M. S. Lubell, R. H. Miller, W. Raith, K. P. Schüler, and J. Sodja, IEEE Trans. Nucl. Sci. NS-24, 1603 (1977).

${ }_{64}$ M. J. Alguard. J. E. Clendenin. P. S. Cooper, R. D. Ehrlich, V. W. Hughes, M. S. Lubell. G. Baum, and K. P. Schüler, Phys. Rev. A 16, 209 (1977).

65 E. H. A. Granneman, M. Klewer, and M. J. van der Wiel, J. Phys. B 9, 2819 (1976).

tit P. J. Keliher, R. E. Gleason, and G. K. Walters, Phys. Rev. A 11, 1279 (1975)

${ }^{67}$ G. Baum, E. Kisker, A. H. Mahan, W. Raith, and B. Reihl, Appl. Phys. 14, 149 (1977)
${ }^{68}$ M. R. O’Neill, M. Kalisvaart, F. B. Dunning, and G. K. Walters, Phys. Rev. Lett. 34, 1167 (1975).

${ }^{69}$ D. T. Pierce, F. Meier, and P. Zucher, Appl. Phys. Lett. 26, $670(1975)$

${ }^{70}$ D. T. Pierce and F. Meier, Phys. Rev. B 13, 5484 (1976)

"C. K. Sinclair (private communication).

${ }^{72} \mathrm{E}$. Reichert (private communication).

73 M. Eminyan (private communication)

${ }^{74}$ E. Garwin, F. Meier, D. T. Pierce, K. Sattler, and H. Ch Siegmann, Nucl. Instrum. Methods 120, 483 (1974).

75 U. Heinzmann, J. Kessler, and J. Lorenz, Z. Phys, 240, 42 (1970)

${ }^{76}$ W. von Drachenfels, U. T. Koch, R. T. Lepper, T. M. Müller, and W. Paul, Z. Phys. 269, 387 (1974).

77 E. H. A. Granneman, Polarization Effects in One-and Two-Photon Ionization of Cesium and Rubidium (academisch proefschrift, University of Amsterdam, 1976, unpublished).

${ }^{8}$ C. K. Sinclair, E. L. Garwin, R. H. Miller, and C. Y. Prescott, in High-Energy Physics with Polarized Beams and Targets, AIP Conf. Proc. No. 35, edited by M. L. Marshak (AIP, New York, 1976), pp. 424-431.

${ }^{79}$ M. J. Seaton, Proc. R. Soc. London A208, 418 (1951).

80 U. Fano, Phys. Rev. 178, 131 (1969)

${ }^{81}$ M. S. Lubell and W. Raith, Phys. Rev. Lett. 23, 211 (1969)

${ }_{82}$ J. Kessler and J. Lorenz, Phys. Rev. Lett. 24, 87 (1970).

${ }_{83}$ U. Heinzmann, J. Kessler, and J. Lorenz, Phys. Rev. Lett. 25, $1325(1970)$

${ }^{* 4}$ G. Baum, M. S. Lubell, and W. Raith, Phys. Rev. Lett. 25, $267(1970)$.

B5 G. Baum, M. S. Lubell, and W. Raith, Phys. Rev. A 5, 1073 (1972).

86 N. F. Mott, Proc. R. Soc. London A124, 425 (1929).

${ }^{87}$ N. F. Mott, Proc. R. Soc. London A135, 429 (1932).

${ }^{8}$ C. G. Shull, C. J. Chase, and F. E. Meyers, Phys. Rev. 63, 29 (1943).

${ }^{84}$ N. Sherman, Phys. Rev. 103, 1601 (1956)

90 L. Mikaelyan, A. Borovoi, and E. Denisov, Nucl. Phys. 47, 328 (1963).

91 J. Van Klinken, Nucl. Phys. 75, 161 (1966).

92 J. S. Greenberg, D. P. Malone, R. L. Gluckstern, and V. W. Hughes, Phys. Rev. 120, 1393 (1960).

93 W. Eckstein, Polarisationseffekte bei der elastischen Elektronenstreuung (Institut für Plasmaphysik, Garching bei München, unpublished).

94 N. Sherman and D. F. Nelson. Phys. Rev. 114, 1541 (1959).

95 G. Holzwarth and H. J. Meister, Nucl. Phys. 59, 56 (1964).

96 Tables of Asymmetry, Cross-section and Related Functions for Mott Scattering of Electrons by Screened Gold and Mercury Nucleii, edited by G. Holzwarth and H. J. Meister (Institut für Theoretische Physik der Universität München, Germany, 1964).

97 Shin-R Lin, Phys. Rev. 133, A965 (1964).

9* W. Bühring, Z. Phys. 212, 61 (1968).

99 V. A. Apalin, I. Ye. Kutikov, J. J. Lukashevich, L. A. Mikaelyan, G. V. Smirnov, and P. Ye. Spivak, Nucl. Phys. 31, 657 (1962).

100 V. Eckardt, A. Ladage, and U. V. Moellendorff, Phys. Lett. 13,53 (1964)

101 J. A. Giordmaine and T. C. Wang, J. Appl. Phys. 31, 463 (1960)

${ }_{102}$ C. B. Lucas, J. Phys. E 6, 991 (1973).

${ }^{103}$ M. J. Copley and T. E. Phipps, Phys. Rev. 48, 960 (1935)

109 W. Schroen, Z. Phys. 176, 237 (1963).

105 W. B. Herrmannsfeldt, SLAC Report No. 166, 1973 (Stanford Linear Accelerator Center, unpublished).

${ }^{106}$ H. D. Zeman, K. Jost, and S. Gilad, Rev. Sci. Instrum 42, 485 (1971).

107 J. W. McGowan and E. M. Clarke, Phys. Rev. 167, 43 (1968).

${ }^{106} \mathrm{H}$. Frauenfelder and A. Rossi, in Methods of Experimental Physics, edited by L. C. L. Yuan and C. S. Wu (Academic, New York. 1963), Vol. 5, Pt. B. pp. 214-274

109 Note that in V. W. Hughes et al., Phys, Rev. A 5, 195 (1972) (Ref. 60), $\alpha$ was erroneously reported as 0.0035 rather than 0.0026

10 U. Heinzmann, J. Kessler, and B. Ohnemus, Phys. Rev. Lett. 27, 1696 (1971).

11 D. Ross, Lasers, Light Amplifiers, and Oscillators (Academic, London, 1969), p. 354

112 G. V. Marr and D. M. Creek. Proc. R. Soc. London A304, $233(1968)$.

113 R. S. Adhav and R. W. Wallace, IEEE J. Quantum Electron. QE-9, 855 (1973)

${ }^{114}$ M. W. Dowley and E. B. Hodges, IEEE J. Quantum Electron. QE-4,

${ }^{15}$ G. E. Francois, Phys. Rev, 143, 597 (1966)

1 th G. D. Bayd and D. A. Kleinman, J. Appl. Phys. 39, 3597 (1968). 